\title{
Non-Single Viewpoint Catadioptric Cameras: Geometry and Analysis
}

\author{
Rahul Swaminathan, Michael D. Grossberg and Shree K. Nayar \\ Department of Computer Science, Columbia University \\ New York, New York 10027 \\ Email: \{srahul, mdog, nayar\}@cs.columbia.edu
}

\begin{abstract}
Conventional vision systems and algorithms assume the camera to have a single viewpoint. However, sensors need not always maintain a single viewpoint. For instance, an incorrectly aligned system could cause non-single viewpoints. Also, systems could be designed to specifically deviate from a single viewpoint to trade-off image characteristics such as resolution and field of view. In these cases, a locus of viewpoints is formed, called a caustic. In this paper, we present an in-depth analysis of the viewpoint loci for catadioptric cameras with conic reflectors. Properties of these viewpoint loci with regard to field of view, resolution and other geometric properties are presented. In addition, we present a simple technique to calibrate such non-single viewpoint catadioptric cameras and estimate their viewpoint loci (caustics) from known camera motion.
\end{abstract}

Index: catadoptric system, conic section, non-single viewpoint, caustics, self-calibration 



\section{Introduction}

Traditionally, imaging systems have been designed to maintain a single viewpoint. In other words, all the rays of light entering the camera intersect at a single point, called the effective pinhole. This pinhole model is extensively used in many vision algorithms. The single viewpoint is not limited to lens based (dioptric) cameras alone. Catadioptric (combination of lens and mirrors) cameras have also been designed with the aim of maintaining a single effective viewpoint.

Single viewpoint catadioptric cameras include multi-sensor planar mirror systems for panoramic imaging [20] as well as for stereo applications [12]. Curved mirrors have also been used in the past. For instance, hyperbolic $[25,28]$ and parabolic $[21,1,24]$ have already been shown to produce single viewpoint catadioptric sensors. These systems consist of a perspective or telecentric ${ }^{1}$ lens and a reflector. The pinhole (entrance pupil) of the lens is positioned at one of the focal points of the reflector, making the other focal point the effective viewpoint. However, such systems require precise assembly of the imaging components, failing which the viewpoint deviates from a single point.

Cameras need not always meet the single viewpoint constraint. It is known that relaxing this constraint, gives greater flexibility in designing imaging systems. Thus, if we allow for deviations from a single viewpoint we could possibly trade-off image characteristics such as field of view and spatial resolution. Catadioptric cameras not having a single view point include: the spherical and conical reflector based designs $[16,27,26,3,19,9,4,10]$. Chahl and Srinivasan developed a specially shaped reflector in [8] so as that the visible solid angle at each pixel was the same. In [15], Hicks and Bajcsy developed a wide angle perspective projection camera system also using specially shaped reflectors. In [22], a conical mirror system was proposed to capture a high resolution $360 \times 360$ degree stereo panorama. All these imaging systems do not maintain a single viewpoint. However, the images acquired by such imaging systems were processed ignoring non-single viewpoint effects. Ideally, the imaging geometry must be understood in order to correctly process the acquired images.

When an imaging system does not maintain a single viewpoint, a locus of viewpoints in three dimensions is formed, called a caustic [5]. For dioptric systems this is termed as a diacaustic and for catadioptric

\footnotetext{
${ }^{1}$ A telecentric lens yields an orthographic projection of the scene onto the image detector.
} 
systems as a catacaustic. The caustic represents the envelope of all incoming scene rays which are eventually imaged. Each pixel in the image maps to a point on the caustic surface. Also, every point on the caustic maps to an unique light ray from the scene which eventually gets imaged. Thus, caustics completely describe the geometry of an imaging system.

Certain applications demand the sensor to have a specific viewpoint surface. In such cases, one can imagine designing specific imaging systems keeping this desired viewpoint locus in mind. For instance, in [1] the caustic was restricted to a single point. In [23] a stereo sensor was designed by constraining the caustic of the camera to be a circle. Also, sensors have been designed to provide near-perspective projection for a given plane in the scene (see[15]).

In this paper, we study caustics of conic catadioptric systems. By this we mean that the profile of the mirror is a conic section. Conic reflector based cameras are widely used in vision as well as in astronomical applications. Also, most surfaces can be locally approximated by quadrics. It is therefore interesting to analyze the viewpoint loci of such imaging systems. In particular we raise the following two questions:

- What happens to the viewpoint locus when a system deviates from a single viewpoint? Note that in spite of using a perspective lens, the catadioptric system need not have a single viewpoint. Instead, the viewpoint locus is described by a three dimensional caustic surface.

- Is there a simple way to estimate the viewpoint loci for such non-single viewpoint systems? Note that in spite of using a perspective lens and reflector, the system as a whole need not maintain a single viewpoint. Calibration entails finding the map between every pixel in the image and its corresponding viewpoint.

We begin by deriving the caustic surface for a family of conic catadioptric systems. By careful parameterization of the reflector profile, we present a simple derivation of the viewpoint locus as a threeparameter family of curves. These caustic surfaces are then analyzed for their effects on resolution, field of view, and geometric singularities. It should be noted that, in our analysis we consider the principal rays passing through the entrance pupil of the camera lens to describe the geometry of the sensor. Sec- 
ondary effects such as blurring (due to bundles of rays) do not interfere with the geometric analysis we present. Finally, we present a simple self-calibration technique to estimate the parameters that describe the caustic surface numerically for a catadioptric camera using known camera motion. Although every pixel has an unique viewpoint, calibration of such non-single viewpoint sensors is reduced to estimating only a handful of parameters.

\section{Caustics: Loci of Viewpoints}

When a light ray interacts with either a reflective or refractive interface, it may bend and thus alter its path. The envelope of these reflected or refracted rays is called the caustic surface [14, 5]. Caustics formed by reflecting elements are called catacaustics and those by refractive elements are called diacaustics. Figure 1 illustrates caustics formed by (a) reflection by metal ring and (b) refraction through a transparent sphere. Near the envelope surface (caustic), the rays of light bunch up together, thus forming bright patterns as seen in the images. Henceforth, we use the term caustic to mean both the catacaustic as well as the diacaustic.

With respect to imaging devices, caustics represent their loci of viewpoints. The single viewpoint is a degenerate case of a point caustic. Each point on the caustic surface represents the three-dimensional position of a viewpoint and its viewing direction. Thus, the caustic completely describes the geometry of the camera. One can represent compound imaging systems consisting of multiple imaging components, such as lenses and reflectors, by simply their effective caustics.

\subsection{Computing the Caustic}

We now study caustics of catadioptric imaging systems consisting of a perspective or telecentric lens and a single reflector whose profile is a conic section. Although, we use the perspective or telecentric (orthographic projection) lens, the imaging system as a whole (including the reflector) need not maintain a single viewpoint.

We first present caustics of rotationally symmetric catadioptric systems, where the entrance pupil of the lens is located along the axis of symmetry of the reflector (see Fig.2) at a distance $d$ from the origin $O$. Telecentric lenses are modeled by taking the limit $d \rightarrow \infty$,i.e. modeling an orthographic projection. 


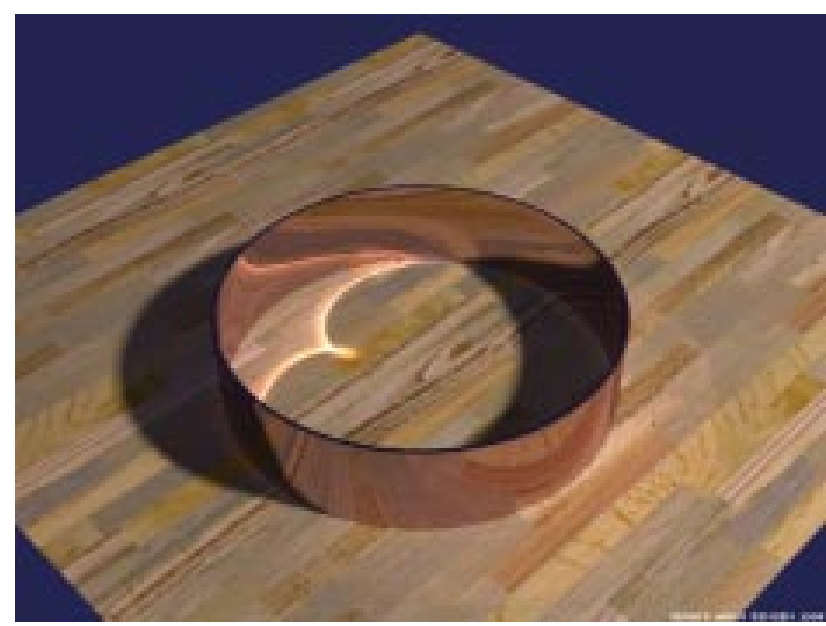

(a)

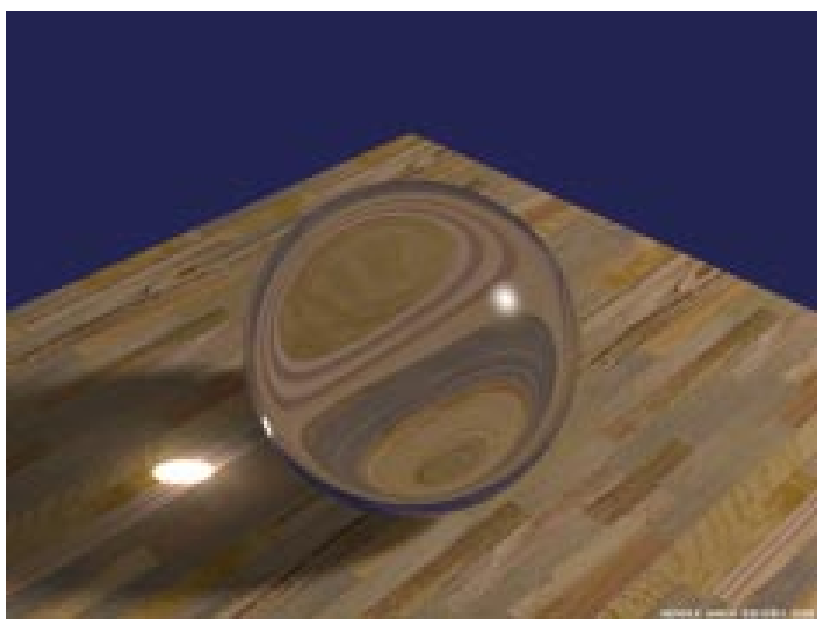

(b)

Figure 1: The caustics formed due to light interacting with (a) a metal ring and (b) a spherical refractive element. Bright patterns of light on the table illustrate a section of the caustic surface formed. These bright patterns are due to the close bunching together of light rays near the caustic's surface. The caustics formed are due to light interacting with (a) a metal ring and (b) a spherical refractive element. Adapted from [17]

The reflector profile is also defined in this coordinate frame. Since the system is rotationally symmetric, analysis is possible in two dimensions (a vertical cross section of the imaging system). However, for asymmetric systems, we will need to derive caustics in three dimensions as shall be shown.

Many techniques have been proposed to derive the caustics of reflecting and refractive systems including ones based on local conic approximations [6] and the Jacobian method [7]. In [6], the central idea lies in fact that for conic sections the light rays emanating from one of the focal points all meet at the second focal point. To estimate the caustic point corresponding to a point on an arbitrary curve, we first approximate the curve locally by a conic section such that one of the foci lies at the entrance pupil of the lens. Thus, the second focal point (derived analytically) is the corresponding point on the caustic curve. However, this approach does not extend to three dimensions as is required in asymmetric systems. We therefore derive the caustic surface based on the Jacobian approach [7], since it easily extends to three dimensions.

In analysing these imaging systems we use the framework of geometric optics. Thus, only the chief rays 


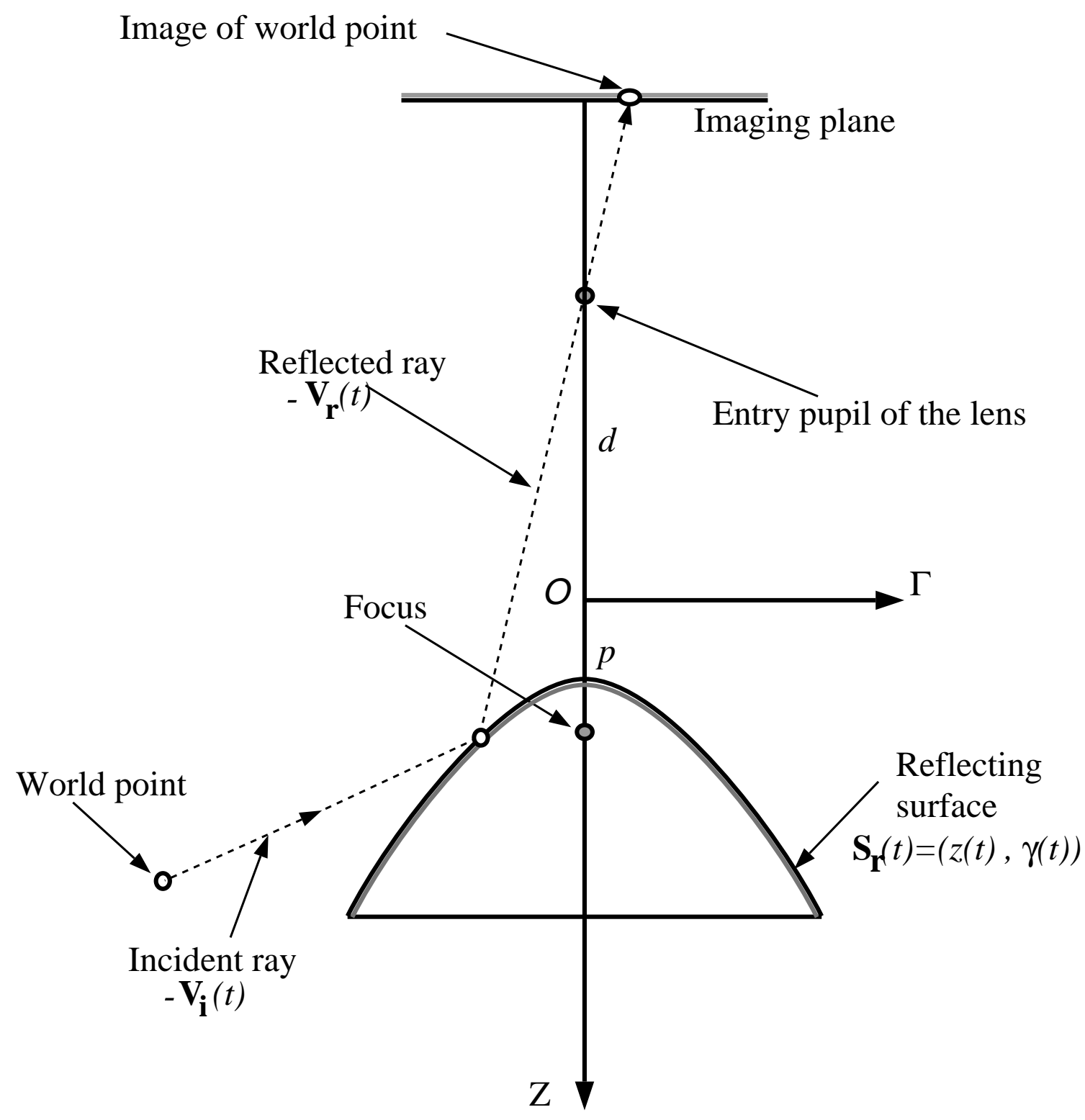

Figure 2: An imaging system consisting of a conic reflector and a perspective lens based camera. The entrance pupil of the lens is located at a distance $d$ from the origin, along the axis of symmetry of the conic section. The reflector profile is also defined in this coordinate frame. A light ray from the scene reflects off the reflector surface and is imaged after passing through the entry pupil. A telecentric lens is modeled by taking the limit $d \rightarrow \infty$. Note that this imaging system can have a non-single viewpoint in spite of using a perspective lens.

or princilpal rays are considered. Although every pixl is associated with a bundle of rays from the scene, only the principal ray are important from a geometric standpoint and will be used in our analysis. In 
contrast, budles of rays are needed to analyse secondary effects such as blurring which is beyond the scope of this paper.

\subsubsection{The Reflector Surface}

Parameterization of the reflector surface is an essential step towards computing the caustic surface analytically. Indeed, whatever the parameterization for the reflector, a solution for the caustic surface exists. We found that standard parameterizations used for conics lead to complicated solutions that are difficult to analyze. In contrast, the following generic parameterization yields a simple solution for the caustic surface. Referring to Fig.2, we define:

$$
\begin{aligned}
& z(t)=t \\
& \gamma(t)=\sqrt{\left(e^{2}-1\right) t^{2}+2 p t-p^{2}}
\end{aligned}
$$

where, $e$ is the eccentricity and $p$ the focus of the conic section. This represents elliptic $(e<1)$, parabolic $(e=1)$ and hyperbolic $(e>1)$ reflectors. The vertex of the reflector is given by $\frac{p}{1+e}$. The $\Gamma$-axis is the directrix of the conic reflector. A point on the reflector surface is then $\mathbf{S}_{\mathbf{r}}(t)=[z(t), \gamma(t)]$. Although, this parameterization has a singularity for spherical reflectors, it makes it easier to analyse and describe the various geometrical properties of the family of viewpoint loci. In Appendix A, we derive caustics using a more generic parameterization which includes the sphere.

\subsubsection{The Rotationally Symmetric Caustic Surface}

From Fig.2, the vector along the reflected ray (entering the lens pupil) is given by:

$$
\mathbf{V}_{\mathbf{r}}(t)=\left[t+d, \sqrt{\left(e^{2}-1\right) t^{2}+2 p t-p^{2}}\right] .
$$

Since we know the geometry of the reflector, its surface normal $\mathbf{N}_{\mathbf{r}}(\mathbf{t})$ can be derived analytically. We then derive the pencil of incident rays $\mathbf{V}_{\mathbf{i}}(t)$ upon reflection as:

$$
\mathbf{V}_{\mathbf{i}}(t)=\mathbf{V}_{\mathbf{r}}(t)-2 \mathbf{N}_{\mathbf{r}}(t)\left(\mathbf{N}_{\mathbf{r}}(t) \cdot \mathbf{V}_{\mathbf{r}}(t)\right)
$$

A point along the incident ray (along $\mathbf{V}_{\mathbf{i}}(t)$ ) parameterized by its distance $r$ from the point of reflection $\mathbf{S}_{\mathbf{r}}(t)$ is then given by: $\mathbf{S}_{\mathbf{r}}(t)+r \cdot \mathbf{V}_{\mathbf{i}}(t)$. The caustic is tangential to the ray along $\mathbf{V}_{\mathbf{i}}(t)$ and hence for 


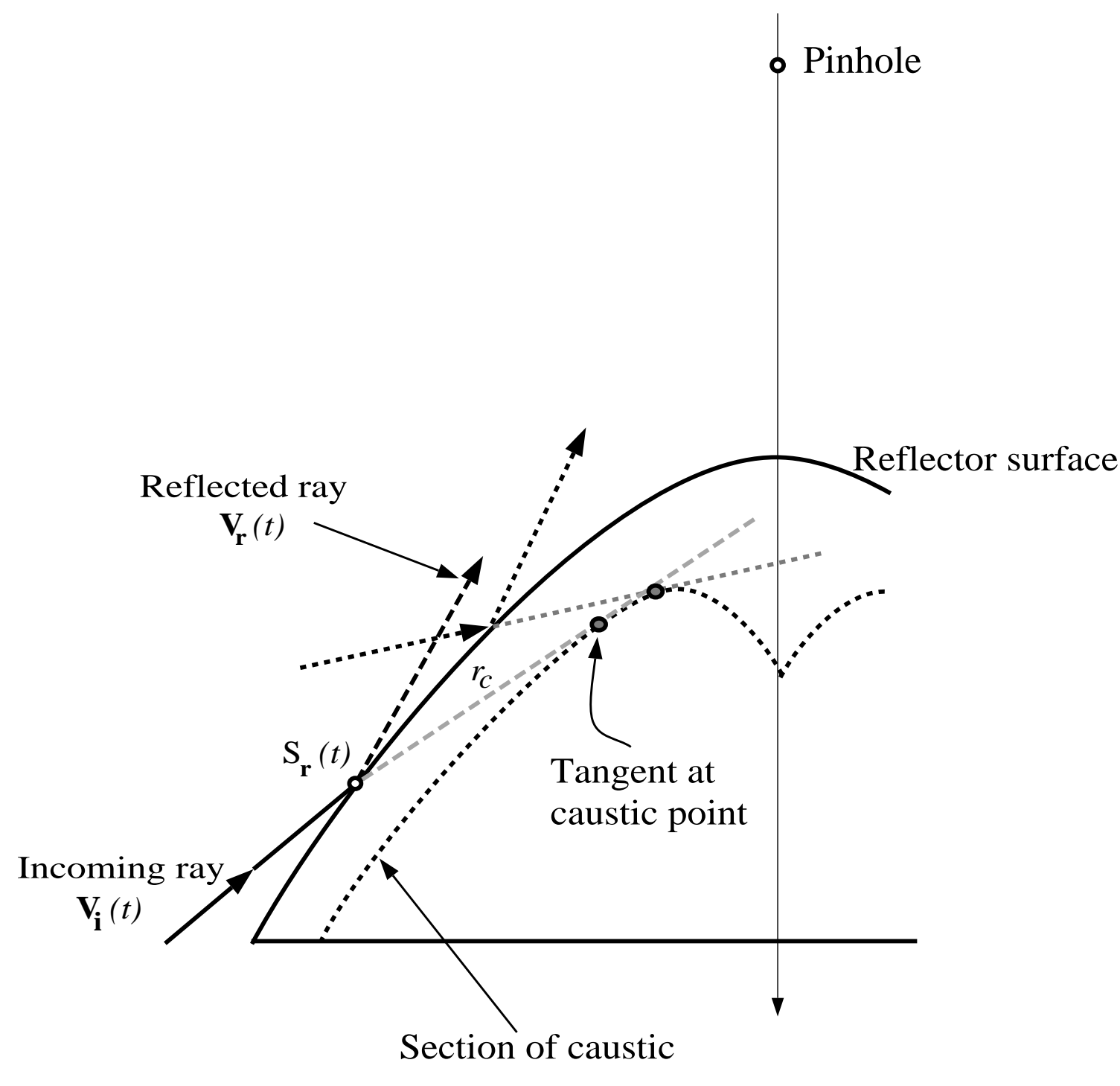

$Z$

Figure 3: Section of the reflector showing incident rays from the scene reflected into the lens. The incident ray $\mathbf{V}_{\mathbf{i}}(t)$ is tangential to the caustic surface. The distance of the point on the caustic from the point of reflection is denoted by $r_{c}$. At the caustic point, if we travel infinitesimally along $\mathbf{V}_{\mathbf{i}}(t)$, we would also move from one ray onto the next. This is due to the close bunching of extended incoming rays at the caustic surface.

some $r_{c}$ the caustic point lies at (see Fig. 3):

$$
\mathbf{S}_{\mathbf{r}}(t)+r_{c} \cdot \mathbf{V}_{\mathbf{i}}(t)
$$


At this point, the light rays bunch up close together to form an envelope. Thus, in the limit, traversing infinitesimally along $\mathbf{V}_{\mathbf{i}}(t)$ at the caustic, is equivalent to traversing from one ray to the next.

Therefore, at the caustic surface, the determinant of the Jacobian $J\left(\mathbf{S}_{\mathbf{r}}(t)+r_{c} \cdot \mathbf{V}_{\mathbf{r}}(t)\right.$ ) must vanish (see [7] for details). Let us now denote the $Z$ and $\Gamma$ components of $\mathbf{S}_{\mathbf{r}}(t)$ by $\mathbf{S}_{\mathbf{r}}(t)_{z}$ and $\mathbf{S}_{\mathbf{r}}(t)_{\gamma}$, respectively, and those of $\mathbf{V}_{\mathbf{i}}(t)$ by $\mathbf{V}_{\mathbf{i}}(t)_{z}$ and $\mathbf{V}_{\mathbf{i}}(t)_{\gamma}$. Enforcing the vanishing constraint on Eq.4 we get:

$$
\operatorname{det}\left(\left[\begin{array}{cc}
\dot{\mathbf{S}}_{\mathbf{r}}(t)_{z}+r_{c} \cdot \dot{\mathbf{V}}_{\mathbf{i}}(t)_{z} & \mathbf{V}_{\mathbf{i}}(t)_{z} \\
\dot{\mathbf{S}}_{\mathbf{r}}(t)_{\gamma}+r_{c} \cdot \dot{\mathbf{V}}_{\mathbf{i}}(t)_{\gamma} & \mathbf{V}_{\mathbf{i}}(t)_{\gamma}
\end{array}\right]\right)=0
$$

where, $\dot{\mathbf{S}}_{\mathbf{r}}(t)=\frac{d \mathbf{S}_{\mathbf{r}}(t)}{d t}$ and $\dot{\mathbf{V}}_{\mathbf{i}}(t)=\frac{d \mathbf{V}_{\mathbf{i}}(t)}{d t}$. Solving for $r_{c}$ we get:

$$
r_{c}(t)=\frac{\dot{\mathbf{S}}_{\mathbf{r}}(t)_{\gamma} \mathbf{V}_{\mathbf{i}}(t)_{z}-\dot{\mathbf{S}}_{\mathbf{r}}(t)_{z} \mathbf{V}_{\mathbf{i}}(t)_{\gamma}}{\dot{\mathbf{V}}_{\mathbf{i}}(t)_{z} \mathbf{V}_{\mathbf{i}}(t)_{\gamma}-\dot{\mathbf{V}}_{\mathbf{i}}(t)_{\gamma} \mathbf{V}_{\mathbf{i}}(t)_{z}}
$$

For the class of conic catadioptric cameras, $r_{c}$ is given by:

$$
r_{c}(t)=\frac{t\left(2 p+t\left(e^{2}-1\right)\right)\left(t\left(d\left(1-e^{2}\right)+p\right)-p(d+p)\right)}{2 p^{2}\left(p^{2}-d^{2}\right)-6 t p^{2}(d+p)-3 t^{2} p(d+p)\left(e^{2}-1\right)-t^{3}\left(e^{2}-1\right)\left(d\left(e^{2}-1\right) d-p\right)} .
$$

Substituting Eq. (7) in Eq. (4), we get the caustic profile for the family of rotationally symmetric conic catadioptric cameras as a three parameter $(e, p, d)$ family of curves:

$$
\begin{aligned}
z_{c}= & \frac{N_{z}}{D_{c}}, \\
\gamma_{c}= & \frac{N \gamma}{D_{c}}, \text { where, } \\
N_{z}= & 2 p^{3}(d+p)^{2}+6\left(d\left(e^{2}-1\right)-p\right) p^{2}(d+p) t \\
& +3 p(d+p)\left(d\left(2-3 e^{2}+e^{4}\right)+2 p\right) t^{2}+ \\
& \left(d^{2}\left(e^{2}-2\right)\left(e^{2}-1\right)^{2}-d\left(4-7 e^{2}+3 e^{4}\right) p\right. \\
& \left.+2\left(e^{4}+e^{2}-1\right) p^{2}\right) t^{3}, \\
N \gamma= & 2(d+p)\left(d-d e^{2}+p+e^{2} p\right) \\
& \left(\left(e^{2}-1\right) t^{2}+2 p t-p^{2}\right)^{\frac{3}{2}}, \\
D_{c}= & e^{2}\left(2(d-p) p^{2}(d+p)+6 p^{2}(d+p) t\right. \\
& +3\left(e^{2}-1\right) p(d+p) t^{2}+\left(e^{2}-1\right) \\
& \left.\left(d\left(e^{2}-1\right)-p\right) t^{3}\right) .
\end{aligned}
$$


The caustic produced due to a telecentric lens and a conic reflector is obtained by taking the limit $d \rightarrow \infty$ :

$$
\begin{aligned}
z_{c}^{\infty}= & \left(2 p^{3}+6\left(e^{2}-1\right) p^{2} t+3\left(2-3 e^{2}+e^{4}\right) p t^{2}+\right. \\
& \left.\left(e^{2}-2\right)\left(e^{2}-1\right)^{2} t^{3}\right) /\left(2 e^{2} p^{2}\right), \\
\gamma_{c}^{\infty}= & \left(\left(1-e^{2}\right)\left(\left(e^{2}-1\right) t^{2}+2 p t-p^{2}\right)^{\frac{3}{2}}\right) /\left(e^{2} p^{2}\right) .
\end{aligned}
$$

We observe from Eq.(8) that the caustic surface is dependent on the distance $d$ of the entry pupil with respect to the reflector, the reflector geometry parameterized by eccentricity $e$, and the focal point $p$. Since conic reflector based catadioptric systems also give single viewpoint imaging systems, we ask: At what distance $d_{0}$ of the entrance pupil would the system produce a point caustic (single viewpoint) at the focus $(p, 0)$ of the reflector? From Eq.(8), we set $z_{c}=p$, and $\gamma_{c}=0$ and solve for $d_{0}$ :

$$
d_{0}=p \frac{e^{2}+1}{e^{2}-1}
$$

In Eq.(10), setting $e=1$ (parabolic reflector) gives $d_{0}=\infty$, implying the use of a telecentric lens as in [21]. Solutions for elliptical $(e<1)$ and hyperbolic $(e>1)$ reflectors suggest the use of perspective lenses located at the focal point of the reflectors. Thus, using the caustic surfaces derived above, we can describe single viewpoint systems as a special case of the general solution.

\subsubsection{The Asymmetric Caustic Surface}

As noted above, when the imaging system is rotationally symmetric, we can compute its caustic surface as a $2 D$ profile. This eases computation and provides a simple solution for the three dimensional surface. However, when the system is not rotationally symmetric, we need to compute the caustic in three dimensions . This occurs when the entrance pupil is not along the axis of the reflector or the reflector itself is not rotationally symmetric. We now present the derivation of the caustic surface for this generic setting.

Referring to Fig.4, let $\mathbf{S}_{\mathbf{r}}(t, u)$ be a point on the three dimensional reflector, parameterized by $(t, u)$. Let $\mathbf{P}$ denote the position of the entrance pupil of the lens. For any such point $\mathbf{S}_{\mathbf{r}}(t, u)$ on the reflector, the ray entering the pinhole can be derived analytically as:

$$
\mathbf{V}_{\mathbf{r}}(t, u)=\mathbf{P}-\mathbf{S}_{\mathbf{r}}(t, u)
$$




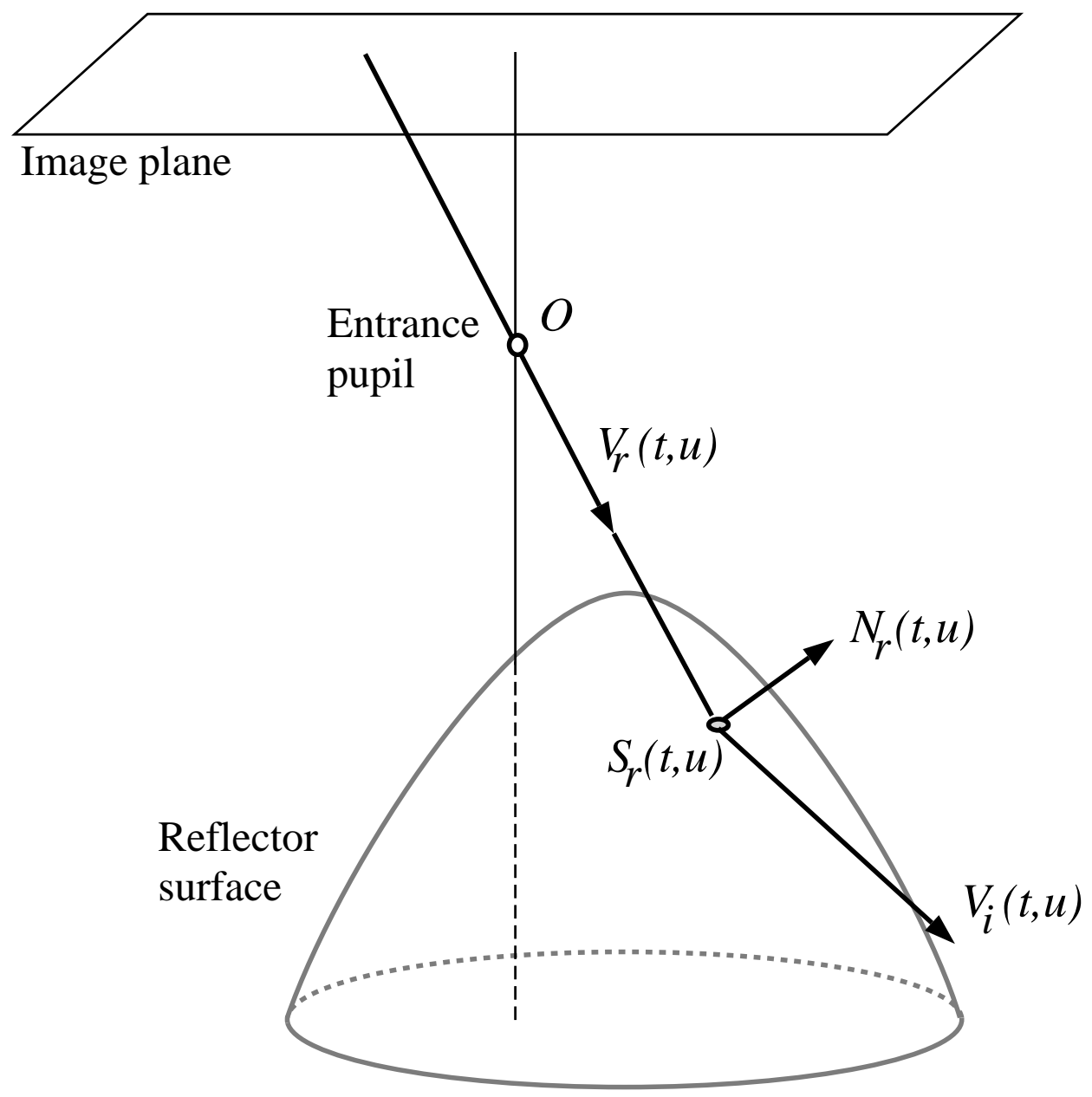

Figure 4: A lens based camera placed off-axis with respect to a rotationally symmetric conic reflector parameterized by $(t, u)$. The viewpoint locus (caustic surface) of such imaging systems is not rotationally symmetric and hence must be derived in three dimensions. The light ray from the scene $V_{i}(t, u)$ reflects off the reflector surface at $\mathbf{S}_{\mathbf{r}}(t, u)$ and is imaged after passing through the entrance pupil $O$ along the reflected ray $V_{r}(t, u)$.

From the known reflector geometry we can derive the vector $\mathbf{V}_{\mathbf{i}}(t, u)$ along the incoming light ray from the reflection equation (similar to Eq.3).

Again, we denote the distance from the point of reflection at which the caustic lies along $\mathbf{V}_{\mathbf{i}}$ by $r_{c}$. The caustic surface then is given by:

$$
\mathbf{S}_{\mathbf{c}}(t, u)=\mathbf{S}_{\mathbf{r}}(t, u)+r_{c} \cdot \mathbf{V}_{\mathbf{i}}(t, u)
$$


Applying the Jacobian method, and the vanishing determinant constraint, we get:

$$
\begin{gathered}
\operatorname{det}\left[J\left(\mathbf{S}_{\mathbf{r}}(t, u)+r_{c} \cdot \mathbf{V}_{\mathbf{i}}(t, u)\right)\right]=0 \\
\operatorname{det}\left(\left[\begin{array}{ccc}
\frac{\partial \mathbf{S}_{\mathbf{r}}(t, u)_{X}}{\partial t}+r_{c} \cdot \frac{\partial \mathbf{V}_{\mathbf{i}}(t, u)_{X}}{\partial t} & \frac{\partial \mathbf{S}_{\mathbf{r}}(t, u)_{X}}{\partial u}+r_{c} \cdot \frac{\partial \mathbf{V}_{\mathbf{i}}(t, u)_{X}}{\partial u} & \mathbf{V}_{\mathbf{i}}(t, u)_{X} \\
\frac{\partial \mathbf{S}_{\mathbf{r}}(t, u)_{Y}}{\partial t}+r_{c} \cdot \frac{\partial \mathbf{V}_{\mathbf{i}}(t, u)_{Y}}{\partial t} & \frac{\partial \mathbf{S}_{\mathbf{r}}(t, u)_{Y}}{\partial u}+r_{c} \cdot \frac{\partial \mathbf{V}_{\mathbf{i}}(t, u)_{Y}}{\partial u} & \mathbf{V}_{\mathbf{i}}(t, u)_{Y} \\
\frac{\partial \mathbf{S}_{\mathbf{r}}(t, u)_{Z}}{\partial t}+r_{c} \cdot \frac{\partial \mathbf{V}_{\mathbf{i}}(t, u)_{Z}}{\partial t} & \frac{\partial \mathbf{S}_{\mathbf{r}}(t, u)_{Z}}{\partial u}+r_{c} \cdot \frac{\partial \mathbf{V}_{\mathbf{i}}(t, u)_{Z}}{\partial u} & \mathbf{V}_{\mathbf{i}}(t, u)_{Z}
\end{array}\right]\right)=0,
\end{gathered}
$$

where the $X, Y$ and $Z$ components of the vectors are denoted as $\mathbf{S}_{\mathbf{r}}(t, u)_{X}, \mathbf{S}_{\mathbf{r}}(t, u)_{Y}$ and $\mathbf{S}_{\mathbf{r}}(t, u)_{Z}$, respectively. Solving the above equation, gives us a quadratic equation in $r_{c}$, the roots of which give us the analytic solution for $r_{c}$. The appropriate root of the equation can be substituted in Eq.(12) to derive the analytic form of the caustic in three dimensions.

\subsection{Examples of Caustic Surfaces}

We now present the viewpoint loci for typical conic catadioptric systems. Figures 5(a), (d) and (g) illustrate viewpoint loci (gray curves) for a catadioptric sensor consisting of a perspective lens and an elliptic, a parabolic and an hyperbolic reflector (dark curves), respectively. The dotted curves in Fig.5(a) denote the part of the elliptic reflector that is self-occluded as well as its corresponding "virtual-caustic". Similarly, Fig.5 (b), (e) and (h) show profiles for catadioptric systems consisting of a telecentric lens and an elliptic, parabolic and hyperbolic reflector, respectively. Note that in Fig.5(e) the caustic degenerates to a point as expected.

Figure 5(c) is a three-dimensional plot of the caustic surface for a symmetric system consisting of a parabolic reflector and a perspective lens. Figure 5(f) depicts the caustic surface in three dimensions for an asymmetric (pupil not on axis of symmetry) catadioptric system. Figure 5(i) is the viewpoint locus for a catadioptric system including a hyperbolic reflector and a telecentric lens. Unlike the other caustic surfaces that are bounded by their reflector's sizes, this caustic surface expands radially.

From the above examples it is clear that the viewpoint locus can be potentially large and at times as large as the reflector itself. To process the image formed by such sensors, one must first understand the geometry of the imaging system. The viewpoint locus derived using the framework of caustics completely describes the geometry of the imaging system. 

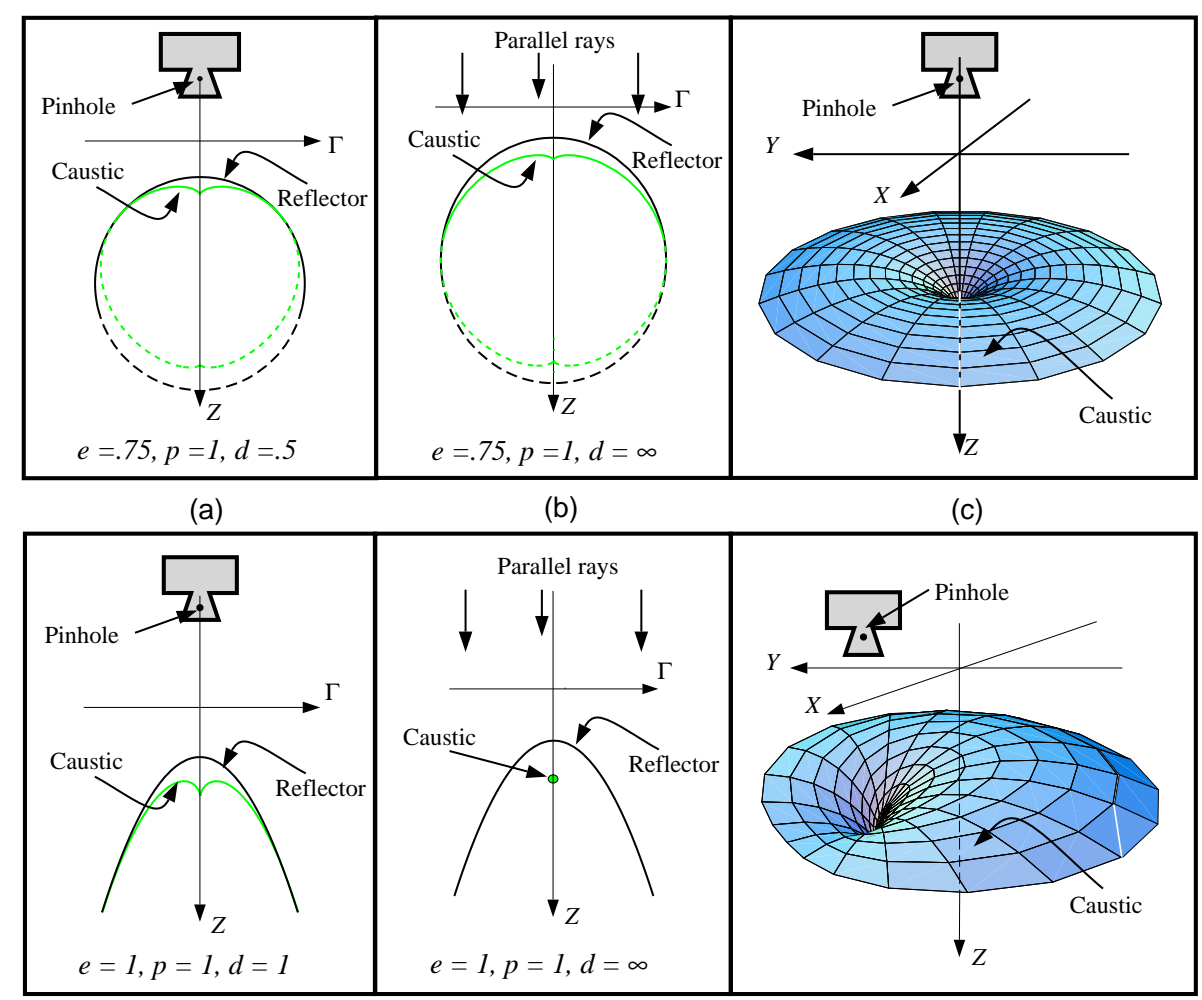

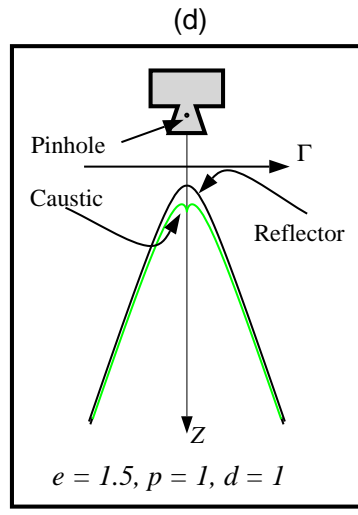

(g) (e)

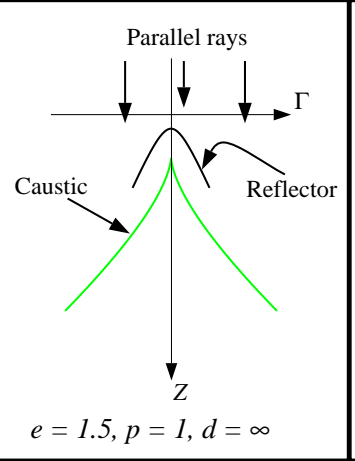

(h) (f)

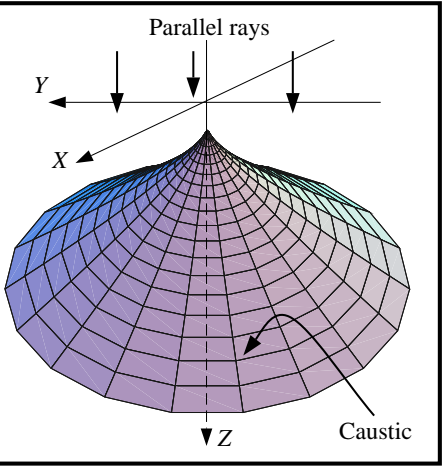

(i)

Figure 5: Viewpoint loci for conic catadioptric systems. Column one: Caustics for symmetric systems consisting of a perspective lens and an (a) elliptic, (d) parabolic and (g) hyperbolic reflector. Column two: Viewpoint loci for catadioptric systems consisting of a telecentric lens and an (b) elliptical, (e) parabolic and (h) hyperbolic reflector. Column three: (c) Caustic surface in $3 D$ for a symmetric catadioptric system consisting of a parabolic reflector and perspective lens. (f) Caustic surface for an asymmetric catadioptric camera consisting of an off-axis perspective lens and a parabolic reflector. (i) Caustic surface for a telecentric and hyperbolic reflector system. All caustics were derived using the Jacobian technique described in Section 2. 


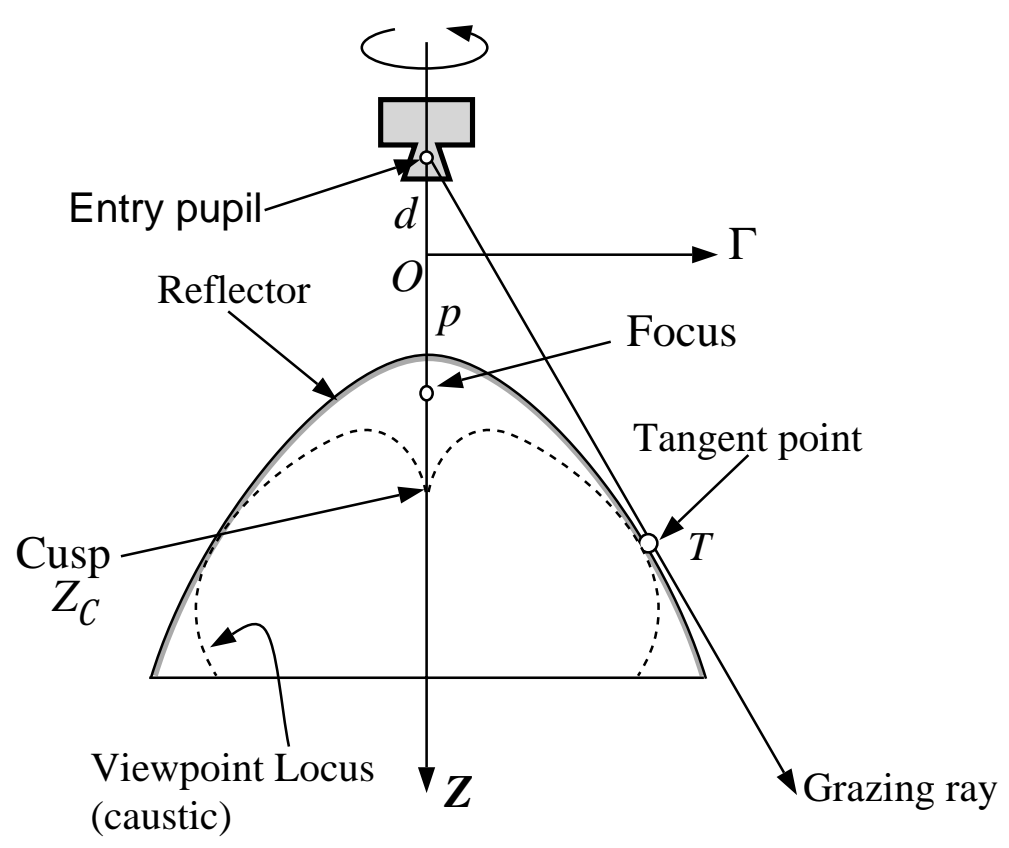

Figure 6: Typical catadioptric camera consisting of a reflector and a perspective lens. The caustic has a singularity denoted by $Z_{\mathcal{C}}$ which we call the cusp. Also shown is the tangent ray to the reflector surface. The point of tangentiality $T$ is also a point on the caustic surface.

\section{Properties of Caustic Surfaces}

We now present some characteristic properties of caustics such as surface singularities and field of view and their relevance to the design of imaging systems. These apply to both convex reflectors and concave reflectors. Most of the observations made are relevant to rotationally symmetric as well as asymmetric case of imaging systems. However, derivations are shown for the rotationally symmetric systems for clarity.

\subsection{Singularities on the Caustic Surface}

As seen from Fig.6, caustic surfaces have singularities which we refer to as cusps. These correspond to the points on the reflector where its surface normal coincides with the reflected light ray (along $\mathbf{V}_{\mathbf{r}}(t, u)$ ) which enters the entrance pupil of the lens (see Fig.6). This constraint in general is given by:

$$
\mathbf{V}_{\mathbf{r}}(t, u)=-\mathbf{N}_{\mathbf{r}}(t, u)
$$


For rotationally symmetric systems with convex or concave reflectors, we get:

$$
\mathbf{V}_{\mathbf{r}}(t)=-\mathbf{N}_{\mathbf{r}}(t)
$$

In such systems, the cusp lies along the optical axis. Referring to Fig.6, we only need to compute the $Z$ coordinate of the cusp. From Eq.(8) and Eq.(14), we solve for the $Z$ coordinate of the cusp:

$$
Z_{\mathcal{C}}=\frac{p\left((1+e)\left(2+e+e^{2}\right) d+2\left(1+e+e^{2}\right) p\right)}{(1+e)\left(2(1+e) d+\left(2+e+e^{2}\right) p\right)}
$$

\subsubsection{The Cusp and Rotationally Symmetric Systems}

We now discuss the significance of the cusp to the design of imaging systems whose caustics are rotationally symmetric. We pose the problem of creating a spherical or cylindrical panorama from the image acquired from a such non-single viewpoint sensor. In such cases, a single center of projection is assumed. Since our sensor does not have a single center of projection, parallax effects are introduced in the panorama. In this setting, how do we measure the degree of parallax errors in the panorama? To measure these errors, we need the location of the virtual center of projection.

We first observe that any incoming light ray, which eventually gets imaged by the detector, meets the optical axis at some point. At the cusp, the light ray passes along the optical axis into the lens. As we move radially outwards, all incoming light rays (tangent to the caustic profile) intersect the optical axis at a point which moves towards the entrance pupil of the lens. Finally, at the very extreme end of the caustic surface, where it touches the reflector, the incoming ray grazes the reflector and meets the optical axis at the entrance pupil. Thus, all the light rays entering the imaging system (tangent to the caustic surface) intersect the optical axis between the entrance pupil and the cusp. The cusp and the entrance pupil therefore bound the possible locations of the virtual center of projection (single viewpoint).

As the entrance pupil moves closer to the reflector, the cusp moves towards the entrance pupil. However,

when the lens moves farther from the reflector, the cusp moves in the opposite direction. In Eq.(15), taking the limit $d \rightarrow \infty$, we find that the position of the cusp converges to:

$$
Z_{\mathcal{C}}^{\infty}=\frac{p(2+e(1+e))}{2(1+e)} .
$$


Thus, even if the exact location of the entry pupil is unknown, one can estimate an upper bound on the cusp's location and still get a good bound on where to approximate the viewpoint locus by a single point.

\subsection{Caustics and Field of View}

Catadioptric systems consisting of convex reflectors have a pencil of rays which graze the reflector surface (see Fig. 7). These rays define the limit of the field of view of the camera. Also, since the caustic touches the reflector at this point, it bounds the size of the caustic surface.

We now show that for reflectors without points of inflection, the point of tangentiality of the grazing ray is also its corresponding caustic point.

Theorem 1 The point on the reflector at which the light ray passing through the lens' entrance pupil pupil grazes the reflector, is also its caustic point.

Proof: Let any point on the reflector be given by $\operatorname{Sr}(x, y)=\{x, y, Z(x, y)\}$. Let the position of the entrance pupil of the lens be given by $\mathbf{P}=\{U, V, W\}$. The light ray entering the pupil is then given by:

$$
\mathbf{V i}(x, y)=\mathbf{S r}(x, y)-\mathbf{P}
$$

For the known reflector, the surface normal is derived as:

$$
\mathbf{N r}(x, y)=\frac{1}{{\sqrt{1+{\frac{\partial Z(x, y)^{2}}{\partial x}}^{2}+\frac{\partial Z(x, y)^{2}}{\partial y}}}^{{ }^{2}}} \cdot\left\{-\frac{\partial Z(x, y)}{\partial x},-\frac{\partial Z(x, y)}{\partial y}, 1\right\}
$$

Since we know that the reflected ray along $\operatorname{Vr}(x, y)$ grazes the reflector surface, it must lie in the plane tangent to $\operatorname{Sr}(x, y)$. We can thus select an appropriate coordinate frame such that the $X Y$-plane corresponds to the tangent plane, giving $\frac{\partial Z(x, y)}{\partial x}=0$ and $\frac{\partial Z(x, y)}{\partial y}=0$. Also, since the pupil $\mathbf{P}$ lies in the $X Y$-plane, $W=0$.

Now using the method described in [7] we compute the desired Jacobian as:

$$
J\left(\operatorname{Sr}(x, y)+r_{c} \cdot \operatorname{Vr}(x, y)\right)=\left[\begin{array}{ccc}
-U & 1+r_{c} & 0 \\
-V & 0 & 1+r_{c} \\
0 & -2 r_{c}\left(V \frac{\partial^{2} Z(x, y)}{\partial x \partial y}+U \frac{\partial^{2} Z(x, y)}{\partial^{2} y}\right) & -2 r_{c}\left(V \frac{\partial^{2} Z(x, y)}{\partial^{y}}+U \frac{\partial^{2} Z(x, y)}{\partial x \partial y}\right)
\end{array}\right]
$$




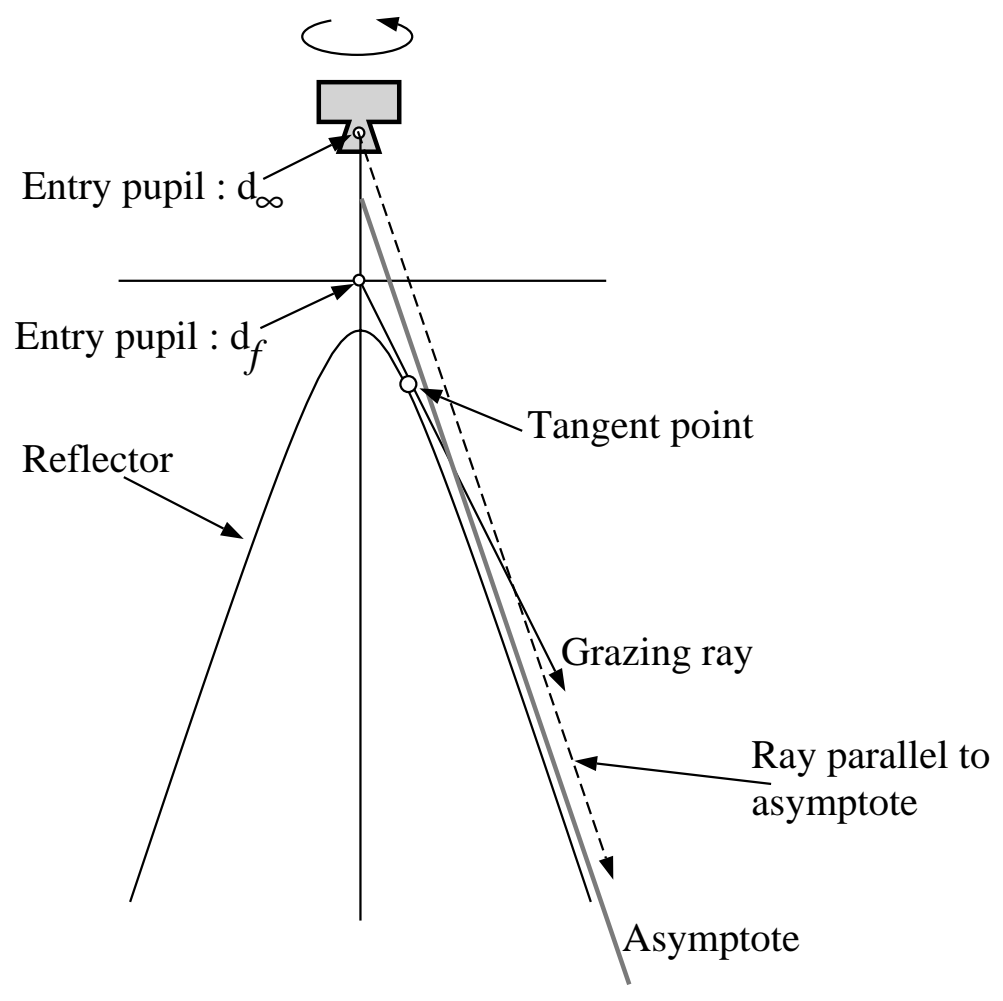

Figure 7: A catadioptric system consisting of an hyperbolic reflector. A entry pupil at $d_{f}$ possesses a ray which is tangential to the hyperbola at a finite point. However, no light ray entering the pupil $d_{\infty}$ can be tangential to the reflector.

Solving for $r_{c}$ (when the Jacobian vanishes) we get:

$$
\begin{aligned}
& r_{c}=0 \\
& r_{c}=1
\end{aligned}
$$

The second solution $r_{c}=1$ is meaningless. However, the right solution is given by $r_{c}=0$.

This proves that the caustic point and the point of tangentiality coincide.

We showed that in three dimensions the grazing ray is tangent to the reflector at a point which is also a point on the caustic surface. A similar result has been already shown for caustics of $2 D$ curves with respect to a point in a plane (see [6]). 


\subsubsection{Location of the Tangent Point}

We now derive the exact parametric form for the location of the "tangent point" using the constraint $r_{c}=0$ for the class of rotationally symmetric conic catadioptric systems. Setting Eq.(6) to be zero, we solve for the point of tangentiality parameterized by $t_{r=0}$ :

$$
t_{r=0}=\frac{p(d+p)}{d+p-d e^{2}}
$$

Amongst the class of conic reflectors, only hyperbolic mirrors do not always have a grazing ray because the reflector is asymptotic. If the entrance pupil of the lens is placed between the vertex of the reflector and the point of intersection between its two asymptotes, a tangent ray (grazing ray) exists which touches the reflector at a finite point. In contrast, if the entrance pupil is located farther away, then there is no tangent ray to the reflector (see Fig.7). The caustic of such an imaging system is also asymptotic, and approaches but never touches the reflector surface.

Due to the non-single viewpoints, perspective (or panoramic) views created would be distorted due to parallax effects. Knowing the point at which the caustic touches the reflector surface, gives us a measure of the caustic's size. This in turn bounds the distortions due to parallax.

\section{Need for computing Caustics in 3D}

In the previous section we claimed that caustics for rotationally asymmetric imaging systems need to be derived in three dimensions. Further, it is impossible to reduce the derivation of the caustic point corresponding to a point on the reflector to two dimensions. We justify these claims by first providing an intuitive reasoning which is later substantiated with a real example.

Referring to Fig. 8, incoming light ray $\mathbf{V}_{\mathbf{i}}(t)$ from the scene lies in the plane $\Pi$ defined by the surface normal $\mathbf{N}_{\mathbf{r}}(t)$ at the point of reflection $\mathbf{S}_{\mathbf{r}}(t)$ and reflected ray $\mathbf{V}_{\mathbf{r}}(t)$ entering the lens. Hence, the caustic point $\mathbf{S}_{\mathbf{c}}$ should also lie in this very plane $\Pi$. Now, the plane $\Pi$ and the reflector intersect to form a planar curve in $3 D$. If two dimensional analysis were sufficient, we should be able to derive the caustic point, from the planar curve, in $\Pi$ alone. However, we further observe that, the surface normals along the curve need not lie in the plane $\Pi$. This non-coplanarity of surface normals entails a complete $3 D$ analysis of 


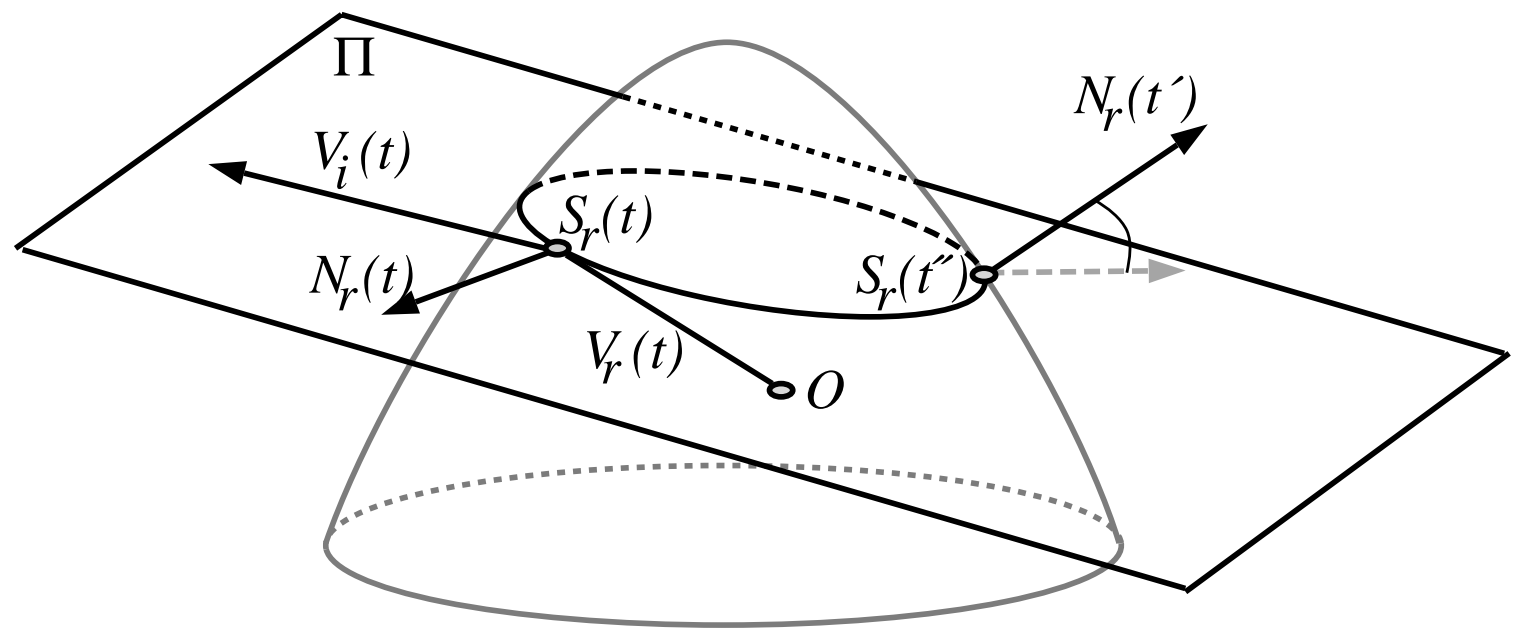

Figure 8: An asymmetric catadioptric camera consisting of a reflector and perspective lens whose entrance pupil is located at $O$. The normal at a point of reflection $\mathbf{S}_{\mathbf{r}}(T)$ and the reflected ray $\mathbf{V}_{\mathbf{r}}$ together define the plane $\Pi$ in which the incident ray $\mathbf{V}_{\mathbf{i}}$ must lie. The plane $\boldsymbol{\Pi}$ intersects the reflector to give us a planar curve in $3 D$. The normal at some other point $\mathbf{S}_{\mathbf{r}}\left(t^{\prime \prime}\right)$ on the reflector along this curve clearly does not lie in the plane $\boldsymbol{\Pi}$. Furthermore, the normal are continuous and thus even in the neighborhood of $\mathbf{S}_{\mathbf{r}}(t)$ the normals are non-coplanar. Thus, solving for the caustic corresponding to $\mathbf{S}_{\mathbf{r}}(t)$ using the planar curve is erroneous.

the caustic surface.

To illustrate this fact, compare the two approaches of deriving the caustic point for a point on a given reflector. If the $3 D$ and $2 D$ analysis were equivalent, then the caustic point should lie at the same distance $r_{c}$ from the reflector in both cases. Thus, comparing the distance $r_{c}$ of the caustic from the point of reflection is sufficient.

Consider a parabolic reflector and a perspective lens in an off-axis configuration as shown in Fig.9 (a). In particular, the reflector surface is defined by:

$$
\mathbf{S}_{\mathbf{r}}(x, y)=\left\{x, y,-\left(z^{2}+y^{2}\right)\right\}
$$

This represents a parabolic reflector, whose vertex lies at the origin. To obtain an asymmetric imaging system, we locate the entrance pupil of the lens at $\{1,0,1\}$ in the $X Z$-plane. This creates an asymmetric viewpoint locus as shown in Fig.9 (b). 


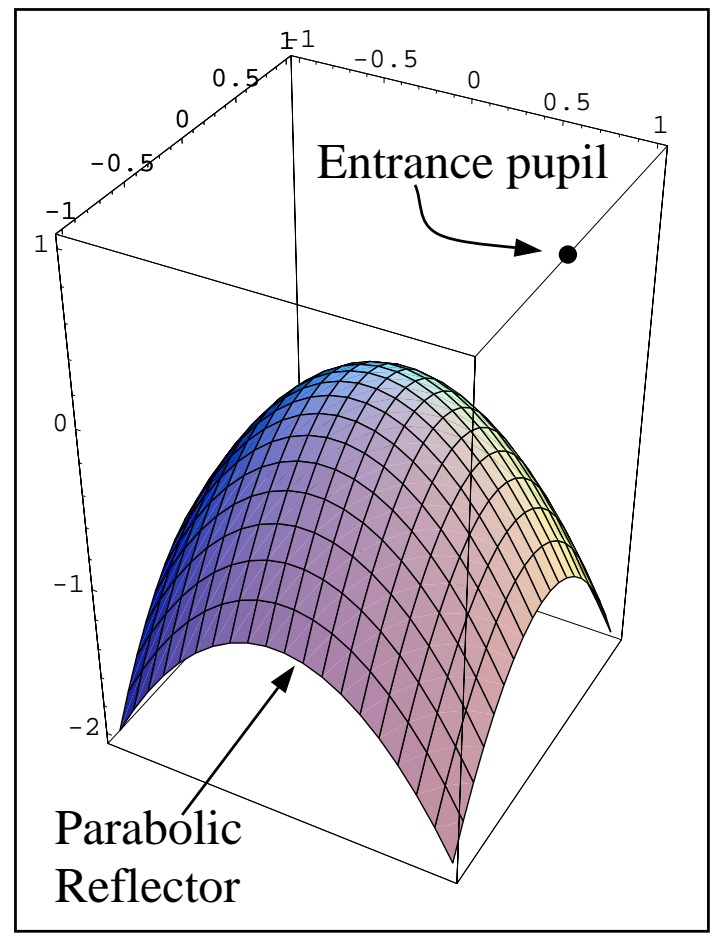

(a)

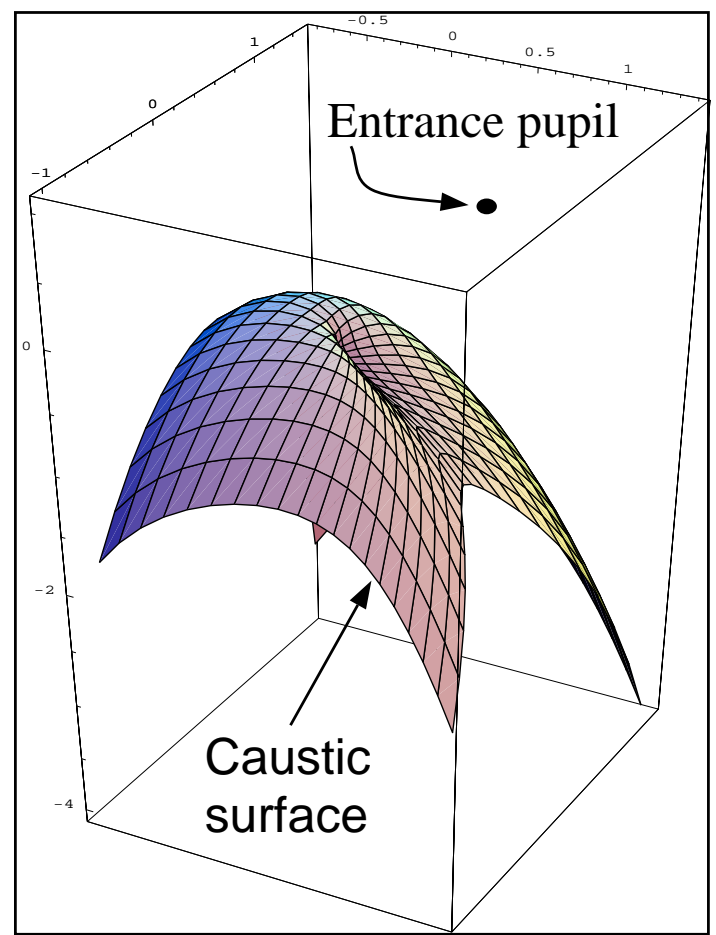

(b)

Figure 9: (a) An asymmetric catadioptric camera consisting of a parabolic reflector and perspective lens whose entrance pupil is located at $\{1,0,1\}$. (b) The corresponding asymmetric viewpoint surface computed using purely three dimensional analysis. Note the peculiar shape and pinching at the cusp.

We now consider a point on the reflector such that the incidence plane does not pass through the axis of symmetry of the reflector ${ }^{2}$. Thus, we wish to compute the caustic corresponding to the point of reflector located at $\mathrm{O}=\{.5, .5,-.5\}$.

\subsubsection{Deriving the Caustic in $3 D$.}

As suggested in $\S 2$, we derive the caustic surface using the vanishing Jacobian constraint[7] (see Fig.9(b)). For the selected point on the reflector $S_{\mathbf{r}}(.5, .5)$, the distance of the caustic point from the point of refection (see Eq. (6)) is computed to be:

$$
r_{c}(.5, .5)=-0.193897
$$

\footnotetext{
${ }^{2}$ It can be shown that if the incidence plane passes through the axis of symmetry, all surface normals for reflector points which are in this plane also lie in the very same plane.
} 


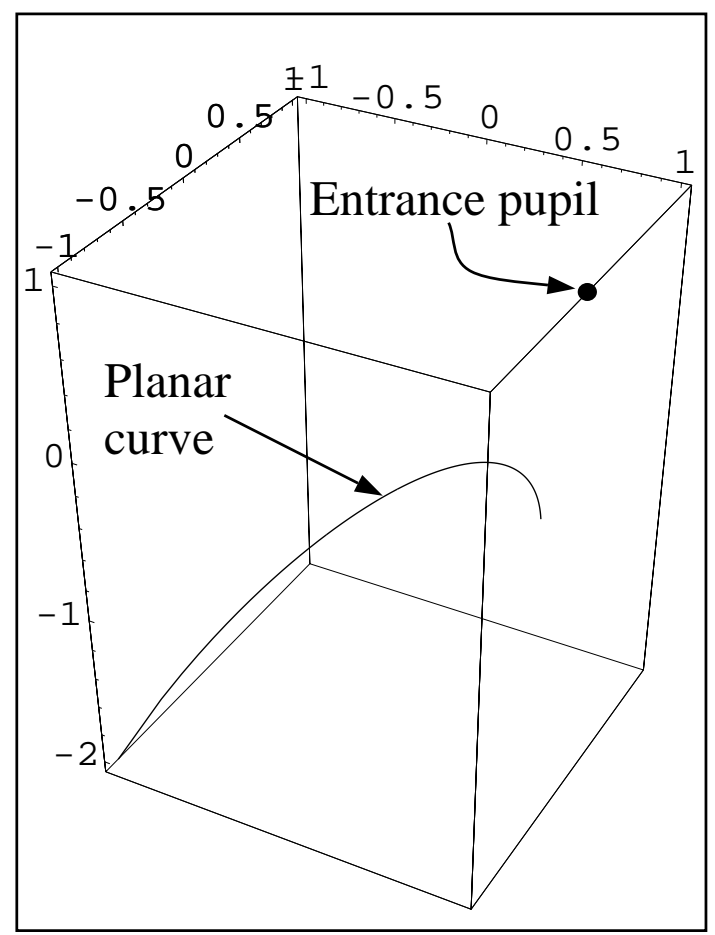

(a)

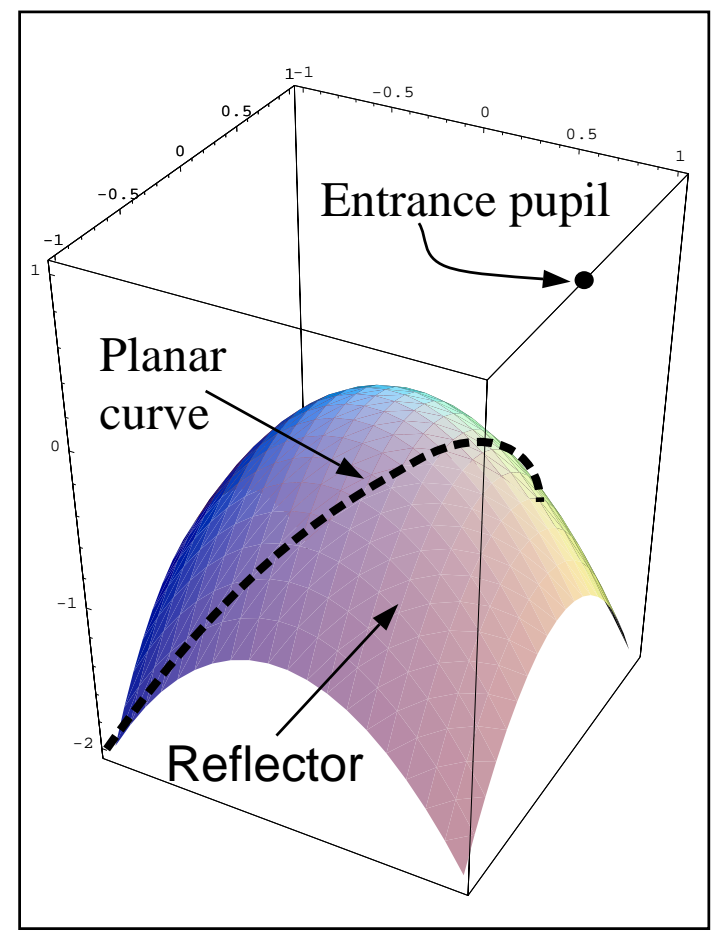

(b)

Figure 10: (a) A section of the planar curve formed by the intersection of the incident plane $\Pi$ and the parabolic reflector. (b) The curve plotted in (a) is superimposed on the reflector to illustrate its exact nature and geometric relationship with the entrance pupil of the perspective lens.

\subsubsection{Deriving the Caustic in $2 D$.}

As discussed earlier, we expect the caustic to lie in the incidence plane $\Pi$ defined by the surface normal at the point of incidence and the reflected ray $\mathbf{V}_{\mathbf{r}}$ entering the entrance pupil of the lens. The incidence plane $\Pi$ and the reflector carve out the planar curve as shown in Figure 10(a-b). For two dimensional analysis, we restrict the caustic computation to this planar curve.

The first step in this process is to derive the planar curve carved by the intersection of the incidence plane $\Pi$ and the reflector. We define the incidence plane $\Pi$ by its normal vector:

$$
\boldsymbol{\Pi}=\mathbf{V}_{\mathbf{r}}(x, y) \times \mathbf{N}_{\mathbf{r}}(x, y)
$$

We now determine the locus of points which lie on the intersection of $\Pi$ and the reflector. Note that the dot product between the vector joining the entrance pupil $O$ and any other point of the surface $\mathbf{S}_{\mathbf{r}}\left(x^{\prime}, y^{\prime}\right)$ 


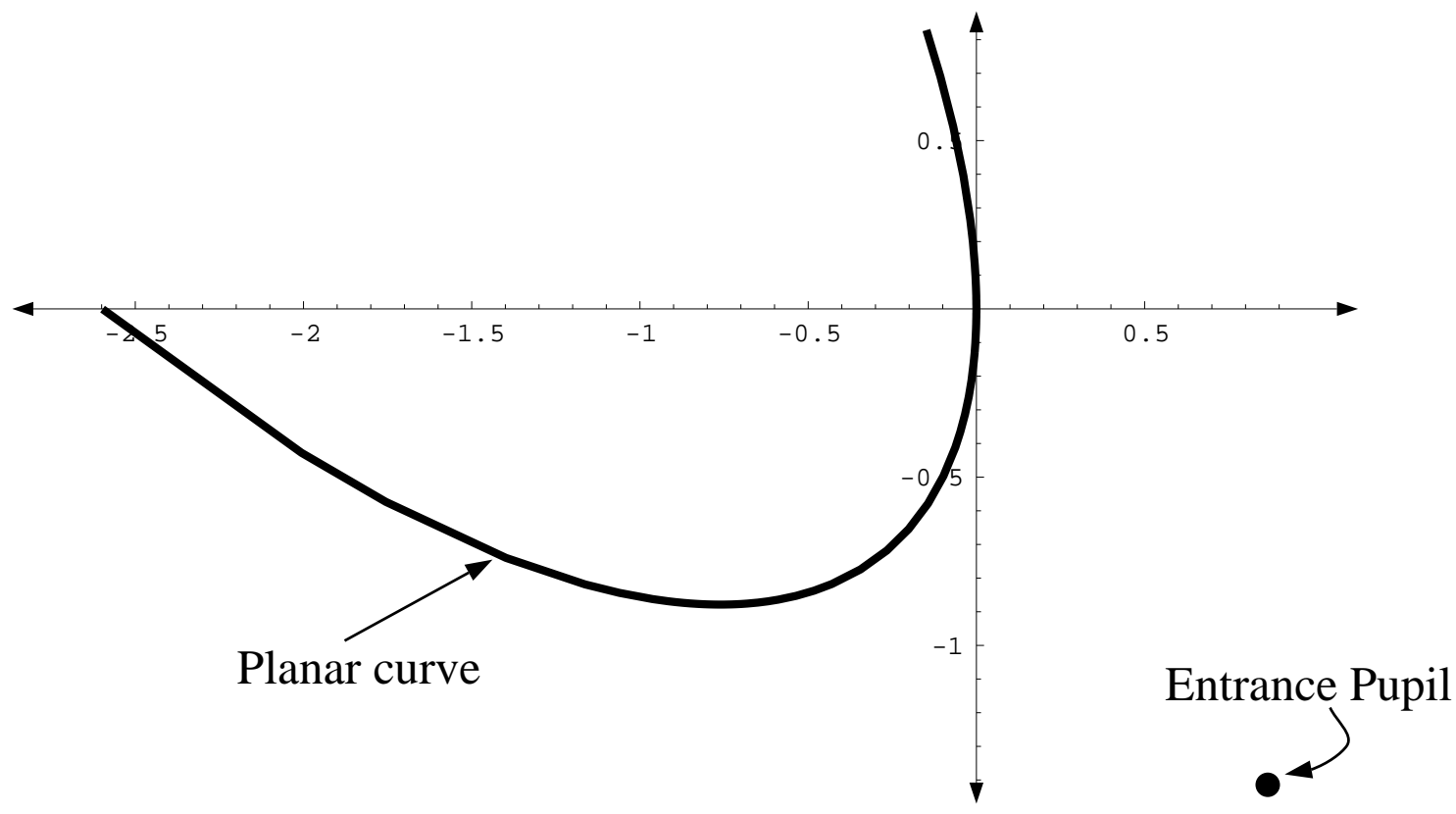

Figure 11: The planar curve (see Fig. 10) was transformed so that, the point of interest on the reflector, namely the point $\{.5, .5,-.5\}$ now lies at the origin and was rotated appropriately so as to completely lie in the $X Z$-plane. Once transformed, we can analyze the caustic of any point on this planar curve simply in the $X Z$-plane. The entrance pupil was also appropriately transformed and is shown as a big black dot.

with the $\Pi$ must vanish. This constraint is given by:

$$
\overrightarrow{\mathbf{S}_{\mathbf{r}}\left(x^{\prime}, y^{\prime}\right)-\mathbf{O}} \cdot \mathbf{\Pi}=0
$$

Solving Eq.(25) for $x^{\prime}$, gives us a parametric form for the planar curve along the reflector of the form (see Figure. 10(a-b)):

$$
\mathbf{S}_{\mathbf{r}}^{\mathbf{2}}\left(y^{\prime}\right)=\left\{x^{\prime}\left(y^{\prime}\right), y^{\prime}, z\left(y^{\prime}\right)\right\}
$$

To simply computing the caustic, we now apply a transformation to the curve as well as the entrance pupil, so as to fix them in the $X, Z$-plane as well as move the point on the reflector to the origin. Figure 11 shows the resulting planar curve and the entrance pupil $\mathrm{O}$ after applying the transformation in the $X Z$-plane.

We now derive the distance of the caustic point corresponding to $\{0,0\}$ using the Jacobian technique for 
two dimensions as:

$$
r_{c}^{2 D}(0)=-0.272784
$$

From Eq. (23) and Eq. (27) it is clear that the distance of the caustic from the reflector surface is different for the two approaches. The two dimensional analysis (in the incidence) plane and the complete three dimensional analysis provide different results. Thus we show by example the need for pure three dimensional analysis when deriving caustics for asymmetric imaging systems.

\section{Resolution}

So far we analysed the geometry of the viewpoint surface, its singularities and other properties. We now analyse the effect of such viewpoint surfaces on resolution characteristics of the sensor. We derive an analytic expression for resolution and show how small design changes impact resolution characteristics dramatically.

In [2], Baker and Nayar showed that conic reflector based single viewpoint catadioptric cameras possess radially increasing resolution. Such imaging systems are a special case of conic catadioptric cameras. We now extend their analysis to include non-single viewpoint imaging systems as well.

\subsection{Single Reflector with Perspective Lens}

Consider an infinitesimal area $\delta A$ in the imaging plane. The angle subtended by this region about the entrance pupil is $\psi$. Let this area image an infinitesimal solid angle $\delta \omega$ of the scene (see Fig. 12). The

resolution of the imaging system is then defined as: $\frac{\delta A}{\delta \omega}$. Thus, smaller the solid angle visible at the infinitesimal region $\delta A$, greater is the resolution.

The solid angle subtended at the entrance pupil of the lens is given by:

$$
\delta \vartheta=\frac{\delta A \operatorname{Cos}^{3}(\psi)}{f^{2}}
$$

where $f$ is the focal length of the lens. The area projected onto the reflector by $\delta A$ is then:

$$
\delta S=\frac{\delta A \operatorname{Cos}(\psi)(d+z(t))^{2}}{\left(f^{2} \operatorname{Cos}(\phi)\right)}
$$




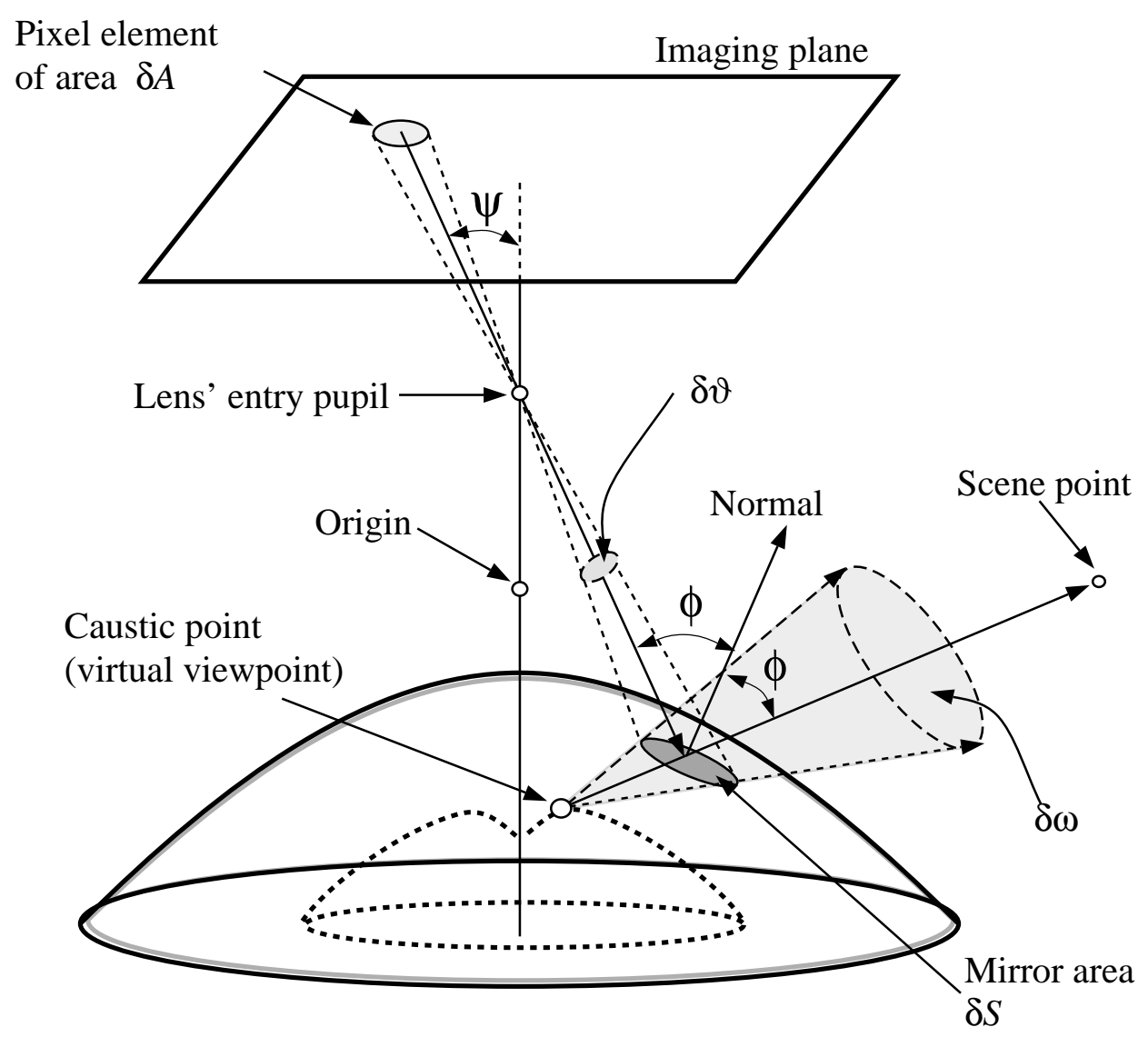

Figure 12: A pixel element of area $\delta A$ in the image plane projects through the entry pupil of the lens onto the reflector as a region of area $\delta S$. The pupil is located at $(0,0, d)$, with respect to the origin. The principal ray from $\delta A$ reflects off the reflector at $s_{R}(x(t, \theta), y(t, \theta), z(t, \theta))$. The corresponding viewpoint on the caustic surface as as shown above. The solid angle subtended at this viewpoint is then $\delta \omega=\delta S / r_{c}^{2}$, where $r_{c}$ is the distance of the viewpoint from the reflector (see Eq.(6)). Resolution is then defined as a ratio of $\delta \omega$ to $\delta A$.

where, $\phi$ is the angle between the principal ray corresponding to $\delta A$ and the surface normal at $\delta S$. The foreshortened area visible to the viewpoint (of the principal ray) on the caustic surface is therefore:

$$
\frac{\delta A \operatorname{Cos}(\psi)(d+z(t))^{2}}{f^{2}} .
$$

The solid angle subtended at this viewpoint is then given by:

$$
\delta \omega=\frac{\delta A \operatorname{Cos}(\psi)(d+z(t))^{2}}{f^{2} \cdot r_{c}(t)^{2}},
$$

where, $r_{c}(t)$ is the distance of the viewpoint from the reflector surface. The resolution for any rotationally 
symmetric catadioptric camera is then given by:

$$
\frac{\delta A}{\delta \omega}=\frac{f^{2} \cdot r_{c}(t)^{2}}{\operatorname{Cos}(\psi)(d+z(t))^{2}} .
$$

Substituting Eq.7 in Eq.32 we obtain the expression for resolution of a rotationally symmetric conic catadioptric system.

We now present the resolution characteristics for some configurations of conic catadioptric cameras. These consist of a conic reflector (whose profile is a conic section) and a perspective lens system, whose entrance pupil is located at a finite distance from the reflector. The resolution results presented are only for rotationally symmetric systems.

Figure 13 illustrates the resolution across a radial slice of the imaging plane. The curves have been normalized with respect to magnification such that area of the reflector's image in all three cases are the same. This facilitates a fair comparison of resolution between the three catadioptric systems. As seen, resolution drops drastically beyond some distance from the image center. This characteristic gradually changes as entry pupil approaches the focal point of the reflector (system becomes single viewpoint). In

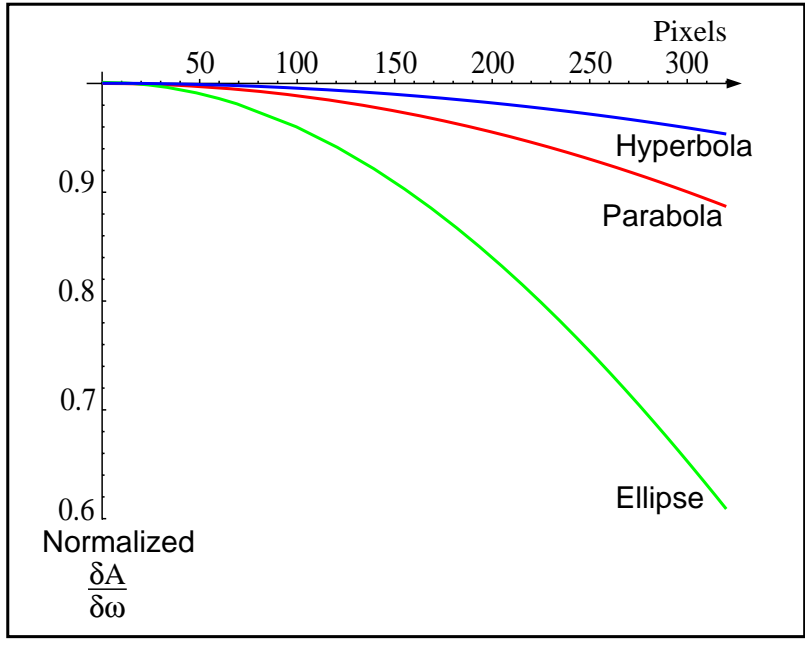

(a)

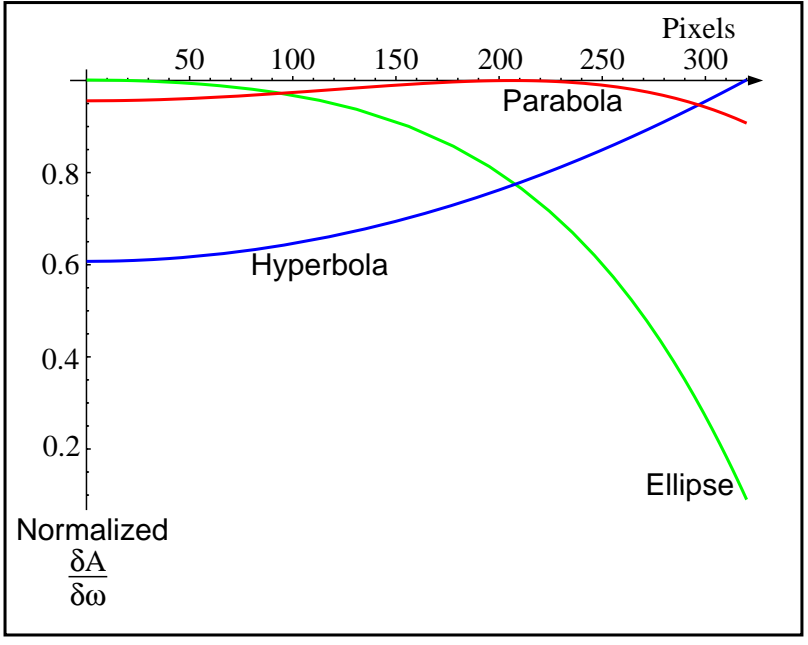

(b)

Figure 13: Plots of resolution for catadioptric sensors having a perspective lens based camera and an elliptic, a parabolic and an hyperbolic reflector. The plots illustrate the resolution across a radial slice of the image plane when the pupil is located at (a) the origin $(d=0)$ and (b) at $d=6$. 
this configuration, resolution increases radially, in keeping with [2].

We now give an intuitive explanation as to why resolution tends to drop towards the edge of the caustic. Typically, the caustic approaches the reflector surface radially outwards. Eventually the caustic touches the reflector at the "tangent point". Thus, $r_{c} \rightarrow 0$ and hence the solid angle subtended increases, reducing resolution. In contrast with single viewpoint systems, $r_{c}$ increases as we move radially outwards, thus increasing resolution.

By careful choice of reflector and positioning of the lens, we can take advantage of the initial rise in resolution (see figure 13(b) ). For instance, by zooming into the reflector (at the cost of reduced field of view) we can use only the section of the reflector for which the optical resolution increases.

\subsection{Single Reflector with Telecentric Lens}

We now discuss a catadioptric imaging system consisting of a telecentric lens and a single conic reflector. From Fig. 14, projection of the area $\delta A$ in the imaging plane onto the reflector is $\delta A / \operatorname{Cos}(\phi)$. The foreshortened patch which is visible from the viewpoint on the caustic surface is $\delta A$. The solid angle subtended at this viewpoint is given by:

$$
\delta \omega^{\infty}=\frac{\delta A}{r_{c}^{\infty 2}},
$$

where $r_{c}$ is the distance of the viewpoint from the point of reflection on the reflector. If $\mathcal{M}$ is the magnification factor, resolution of such a sensor is given by:

$$
\frac{\delta A}{\delta \omega^{\infty}}=\mathcal{M} \cdot r_{c}^{\infty}
$$

For conic reflectors, the distance of the viewpoint from the point of reflection is given by:

$$
r_{c}^{\infty}=-\frac{\left(t\left(p+\left(-1+e^{2}\right) t\right)\left(2 p+\left(-1+e^{2}\right) t\right)\right)}{\left(2 p^{2}\right)} .
$$

Then, resolution for a conic catadioptric imaging system with a telecentric lens is:

$$
\frac{\delta A}{\delta \omega^{\infty}}=\mathcal{M} \cdot \frac{\left(t\left(p+\left(-1+e^{2}\right) t\right)\left(2 p+\left(-1+e^{2}\right) t\right)\right)^{2}}{4 p^{4}} .
$$

We now apply results derived in Eq.(36) to conic catadioptric cameras fitted with a telecentric lens and study their resolution characteristics(see Fig. 15). 


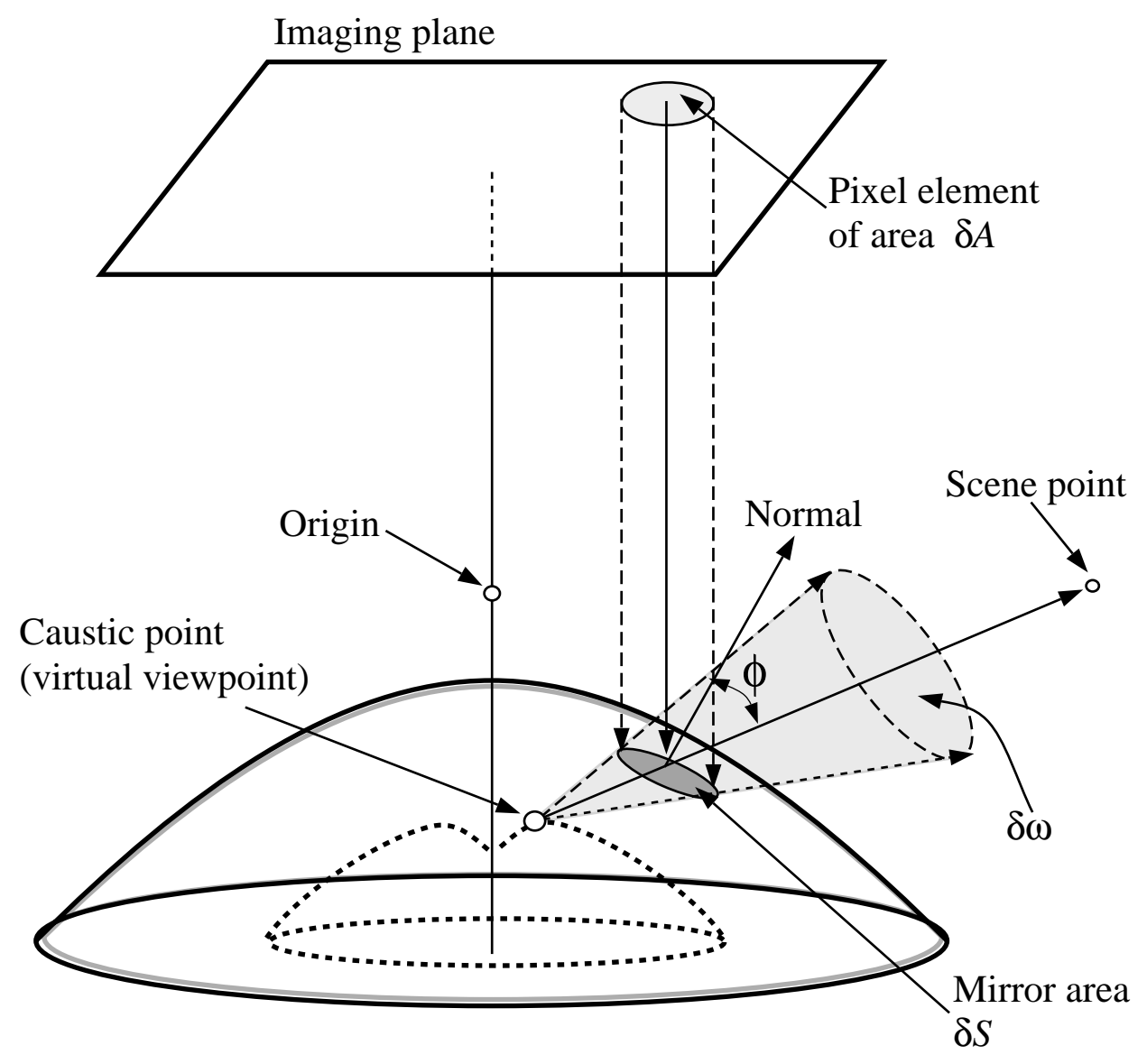

Figure 14: A pixel element of area $\delta A$ in the image plane projects through the telecentric lens (orthographic projection) onto the reflector as a region of area $\delta S$. The principal ray from $\delta A$ (corresponding to the center of the area $\delta A$ ) reflects off the reflector at $s_{R}(x(t, \theta), y(t, \theta), z(t, \theta))$. The corresponding viewpoint on the caustic surface is as shown above. The solid angle subtended at this viewpoint is then $\delta \omega=\delta S / r_{c}^{2}$, where $r_{c}$ is the distance of the viewpoint from the reflector (see Eq.(6)). Resolution is then defined as a ratio of $\delta \omega$ to $\delta A$.

For parabolic reflectors, the caustic reduces to a single point (see [1, 2]). Thus, the resolution increases radially as expected. The caustic surface for an elliptical reflector with telecentric lens is similar in nature to that with a perspective lens. Hence, the resolution characteristics are also similar, exhibiting an initial rise and then a drop towards the periphery. Finally, the hyperbolic reflector yields a caustic which is expanding in nature (see figure 5 (i) ). Also, the direction of expansion is in the direction opposite to that of the point of reflection. This results in radially increasing resolution. 


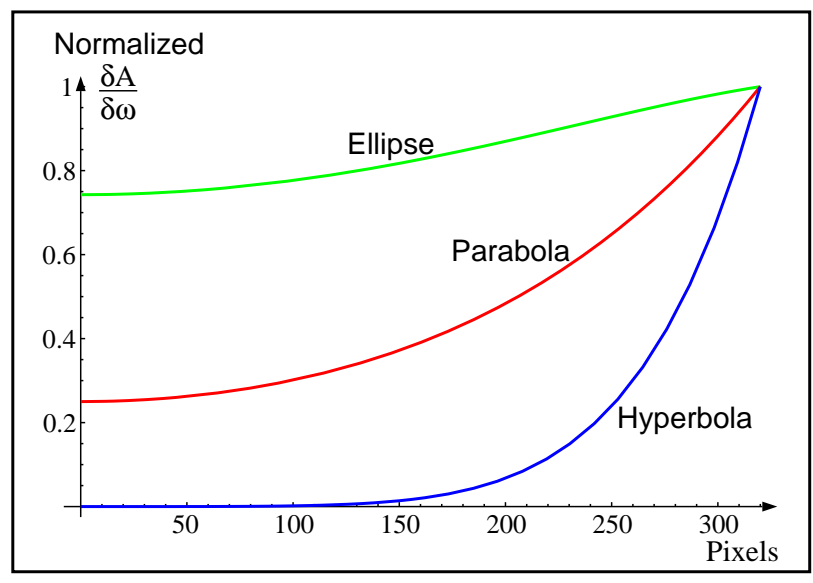

(a)

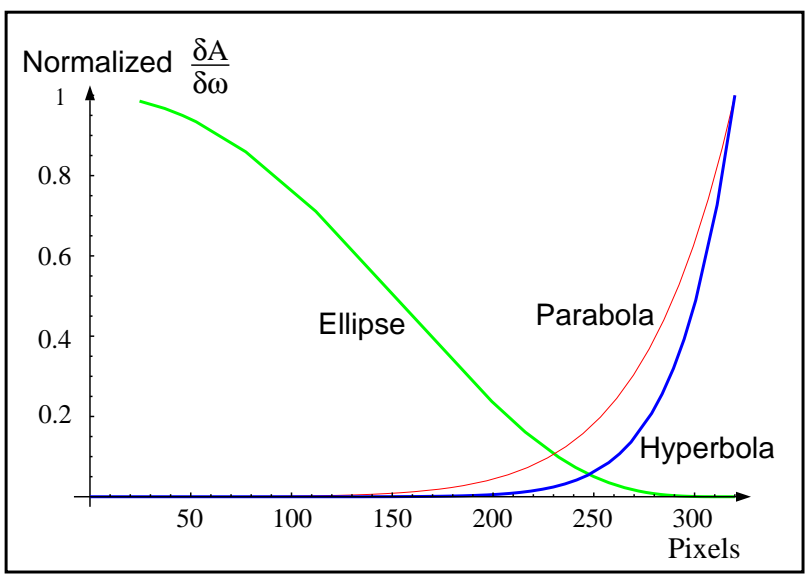

(b)

Figure 15: (a) Resolution across a radial slice of the image plane by a catadioptric sensor consisting of a telecentric lens and a parabolic, elliptic and hyperbolic reflector respectively. The resolution plots have been normalized to illustrate the manner in which resolution changes across the image plane. (b) For elliptic reflector based catadioptric cameras, the resolution initially rises to a maxima then drops to zero at the periphery of the field of view. This drop to zero corresponds to the point on the reflector at which the caustic surface touches it (see Eq.(21)). However, this is not the case with parabolic or hyperbolic reflectors.

\subsection{Designing Resolution Specific Sensors.}

The analytic expressions for resolution of catadioptric cameras are not restricted to conic reflectors alone. In general, the same framework can be extended to any mirror shape. Moreover, the formulae derived above not only aid in the analyzing the resolution of the sensor, but can also be used to drive its design. Consider the class of conic reflector based catadioptric systems. Resolution is parameterized by the geometry of the reflector, the location of the entrance pupil and the focal length of the lens used. Given an apriory resolution curve, we can "fit" the right parameters in the model $(e, p, d, f)$, that most closely approximate the required curve. In the most generic setting, we could let resolution characteristics completely dictate reflector's shape (not restricted to conic reflectors). It should however be noted that by fixing resolution the sensor may not maintain a single viewpoint. Depending on the application at hand, this may or may not be critical. 


\section{Self Calibration of Non-Single Viewpoint Cameras.}

So far we discussed the geometry and resolution characteristics of conic catadioptric cameras. We derived a parametric expression for their viewpoint loci in terms of the geometry and location of the various imaging components. If the exact geometry and configuration of the imaging components such as lenses and mirrors is known, the caustic or viewpoint locus can be derived analytically. However, when these parameters are unknown, self-calibration is required. Although every pixel has an unique viewpoint and viewing direction, the caustic formulation reduces the complexity of calibration to that of estimating only a handful of parameters.

In the past, techniques for calibrating single viewpoint catadioptric systems have been suggested using a single image [11] or camera motion [18]. Recently, Grossberg and Nayar suggest using caustics to represent generic imaging systems, and also presented a calibration technique which requires known motion as well as an active display of known light patterns (see [13]). In contrast, our technique calibrates non-single viewpoint systems having a parametric form for the caustic, using only known camera motion and point correspondences between views of unknown scene points by estimating the model parameters.

\subsection{Objective Function Formulation.}

We pose the caustic estimation problem as one of error minimization. As shown in Fig. 16, let $p_{1}$ and $p_{2}$ be the images of a static scene point $P$, in the two views. From the hypothesized parameter values (during search), we map $p_{1}$ and $p_{2}$ to their corresponding viewpoints $\left(\mathbf{S}_{\mathbf{1}}\right.$ and $\left.\mathbf{S}_{\mathbf{2}}\right)$ as well as their viewing directions $\left(\mathbf{V}_{1}\right.$ and $\left.\mathbf{V}_{2}\right)$. Camera motion is not the only factor that contributes to a new viewpoint. Since the image is formed by reflection off a curved surface, the second view is reflected off a different point on the reflector. This maps to a different position on the caustic as illustrated in Fig.16. Since the two light rays come from the same scene point, they must intersect at the scene point. This happens when the hypothesised caustic parameters match that of the system.

In general we can define the objective function as the distance between these to rays. However, we found it more stable to define the error function in the image space. To do so, we first derive in closed form, a point $\tilde{P}$ along $\mathbf{V}_{1}$ which is closest to ray $\mathbf{V}_{\mathbf{2}}$ (this is the best estimate of the scene point $P$ ). This 


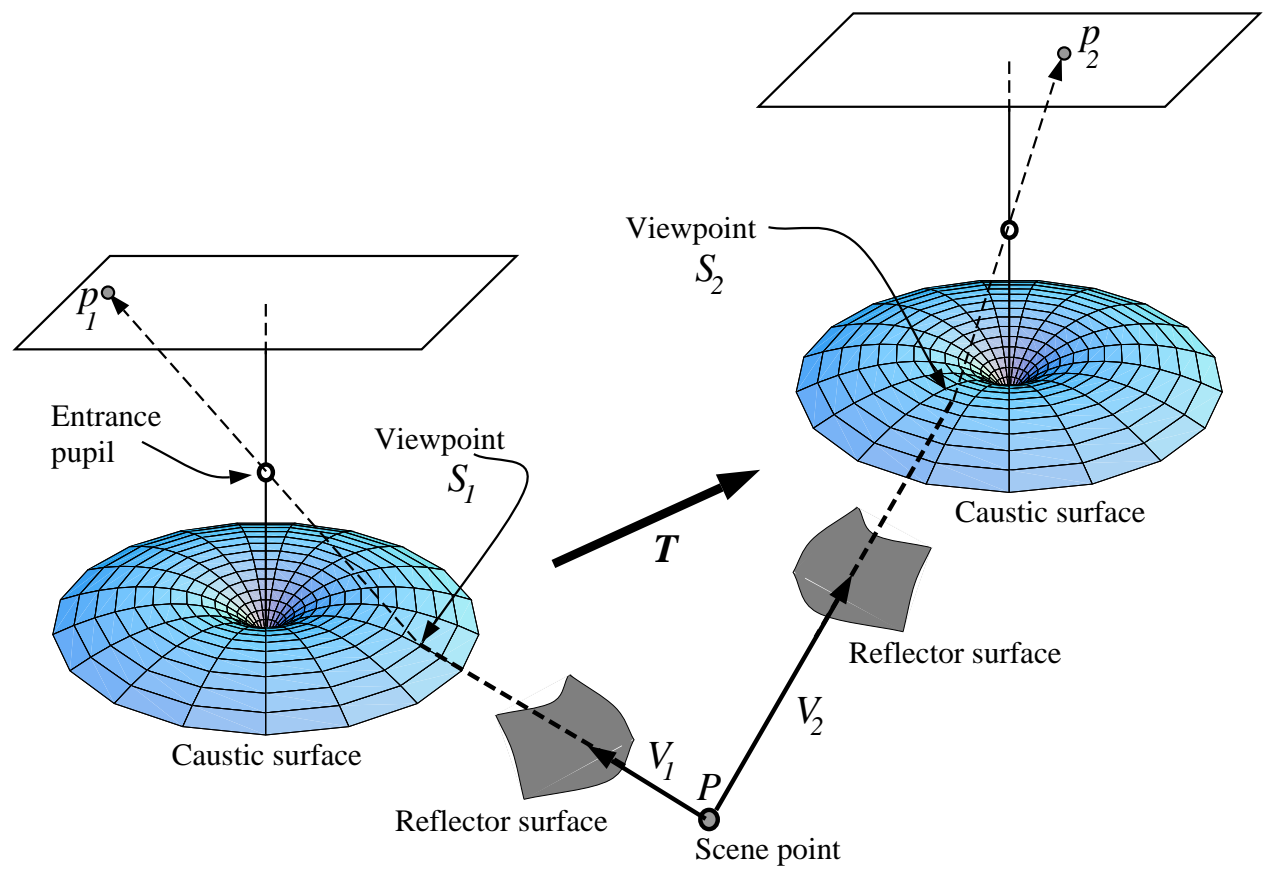

Figure 16: The catadioptric imaging system is represented here by only its caustic surface (viewpoint locus). The two caustic surfaces denote the same camera after being given a known translation $\mathbf{T}$. An unknown scene point $P$ visible from both views gets imaged at two point $p_{1}$ and $p_{2}$ respectively. From the caustic model and the hypothesized parameters during search, we map these image point to their respective viewpoint $S_{1}$ and $S_{2}$ and their viewing directions $V_{1}$ and $V_{2}$. When the right model parameters are estimated, the two position vectors (light rays at viewpoints) must meet at the scene point.

hypothesized scene point $\tilde{P}$ is then mapped onto the image plane in view two, using the caustic model. We then define the error as the disparity between the image of $\tilde{P}$ in the second view $\tilde{p}_{2}$ and known image in the $p_{2}$ :

$$
\epsilon=\left|\tilde{p}_{2}-p_{2}\right|_{2}
$$

The objective function is defined over all the pairs of point correspondences between the two views. Thus, if $p_{i}{ }^{j}$ refers to the $i^{t h}$ image point in view $j$, then the objective function for $N$ scene points is given by:

$$
\xi=\sum_{\forall i \in N}\left|\tilde{p}_{2}^{i}-p_{2}{ }^{i}\right|_{2}
$$




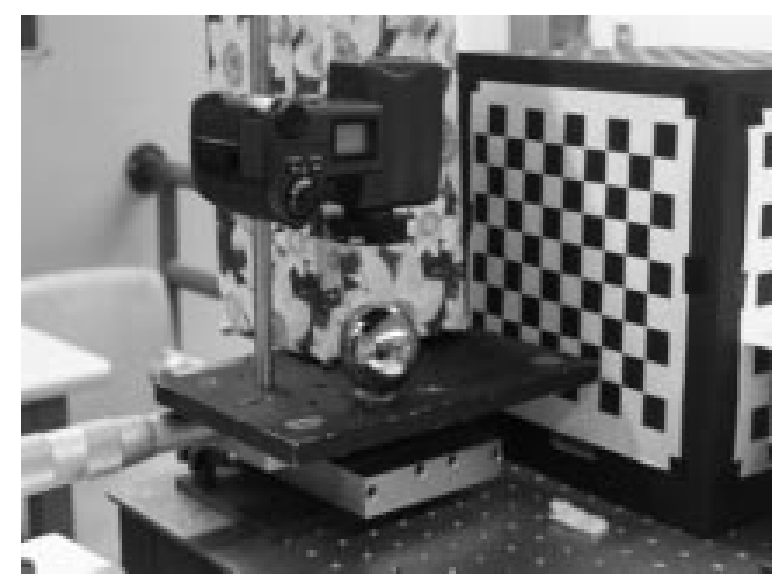

(a)

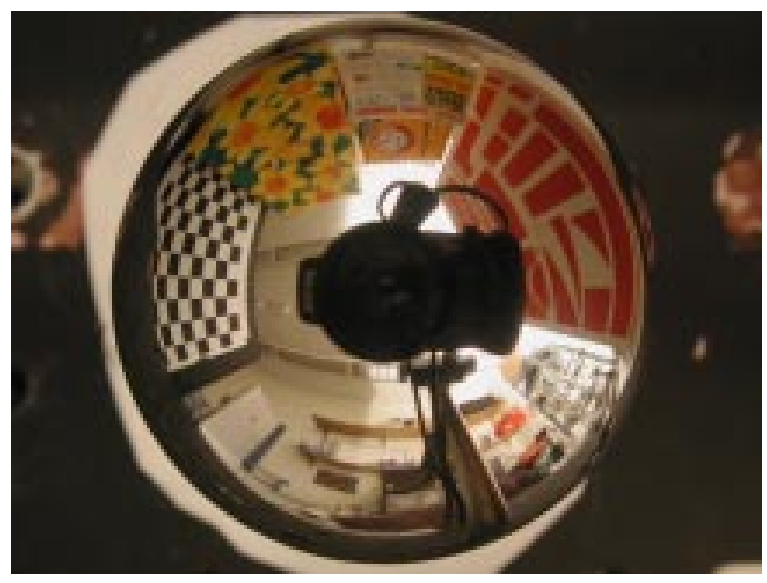

(b)

Figure 17: (a) A catadioptric camera consisting of a perspective lens based digital camera and a spherical reflector (ball-bearing). The reflector's radius is $25.4 \mathrm{~mm}$, and lens' entry pupil is approximately $150 \mathrm{~mm}$ above the center of the sphere. The catadioptric camera was placed on a translation stage and translated by $20 \mathrm{~mm}$ along the $Y$ axis. (b) Sample image acquired during the calibration process. Each image is $2048 \times 1536$ in size.

\subsection{Calibrating a Real Sensor}

We now present calibration results on a real non-single viewpoint catadioptric sensor. The sensor consists of a perspective lens based high resolution digital camera and a spherical reflector (ball bearing). The reflector was two inches in diameter and was placed approximately $150 \mathrm{~mm}$ below the digital camera (see Fig. 17(a)).

The catadioptric camera was placed rigidly on a translation stage to facilitate accurate camera motions. Between acquiring the two views, the sensor was translated precisely by $20 \mathrm{~mm}$ sideways (along the $Y$ axis). Figure 17(b) shows a typical image acquired by this catadioptric sensor.

Since the parameterization in Section 2 is singular for a sphere, we parameterize the reflector surface as:

$$
A \cdot z^{2}+\gamma^{2}+B \cdot z=C
$$

which includes the entire class of conic reflectors. As before, the entrance pupil is at a distance $d$ from the origin along the $Z$ axis. The caustic surface is then described by a four-parameter $(A, B, C, d)$ family of curves (see Appendix A). 


\begin{tabular}{|c|c|c|c|}
\hline & $\sqrt{C}(\mathrm{~mm})$ & $d(\mathrm{~mm})$ & $f$ (pixels) \\
\hline Ground Truth & 25.4 & 150 & 5381 \\
\hline Estimated Values & 24.7 & 176.9 & 5379 \\
\hline
\end{tabular}

Figure 18: Estimated and ground truth parameters for the catadioptric camera shown in Fig.17. The estimation was done using constrained minimization routines in Matlab.

Since the algorithm depends on the non-single viewpoints to estimate the model parameters, it is imperative that the chosen point correspondences come from near by scene points. Just like in stereo, with greater depth, parallax effects diminish, thus minimizing image disparity produced by the the viewpoint locus. In our experiment we used 41 scene points (image correspondences) to guide the non-linear search. Since we know the reflector to be spherical we only need to estimate the focal length $f$ of the perspective lens, the distance $d$ of the lens's entrance pupil from the reflector, and the radius of the reflector $\sqrt{C}$.

Figure 18 provides a comparison between the estimated model parameters and ground truth. The ground truth is based on careful manual measurement of the required parameters. The recovered radius of the reflector is accurate to within a few millimeters. The estimated focal length too is close to the ground

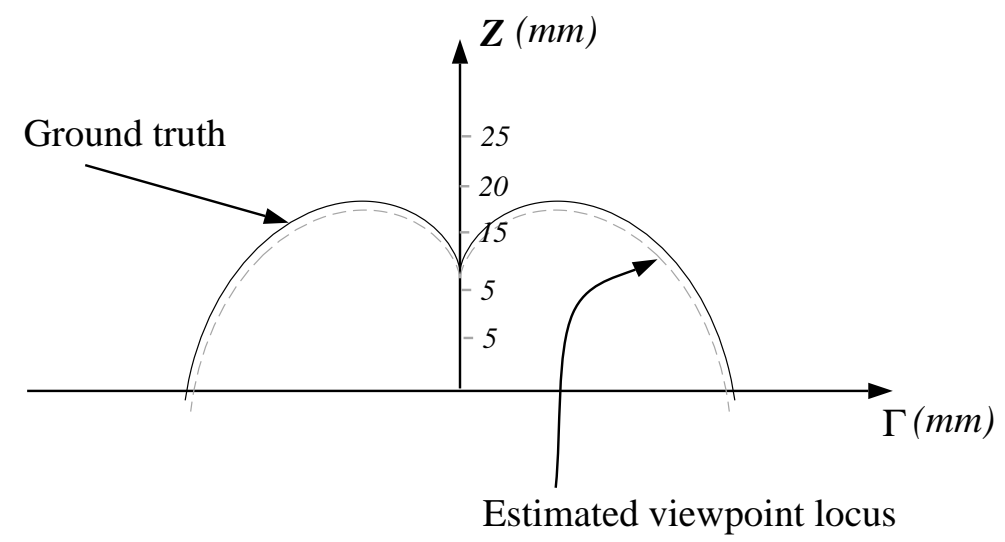

Figure 19: Ground truth (solid curve) and estimated caustic (dotted curve) for the imaging system shown in Fig.17. As seen, the error in the estimated viewpoint locus of the camera is marginal and follows the ground truth caustic closely. 
truth. However, the estimate of $d$ is a little erroneous. This can be either due to incorrect convergence of the search or from erroneous measurement of ground truth. Once the model parameters are estimated we can plot the viewpoint locus for the estimated caustic profile (dotted curve) against ground truth (solid curve) (see Fig. 19).

\section{Summary}

For a while it has been considered necessary for imaging sensors to maintain a single viewpoint. In reality this limits the flexibility on has in designing optical systems. For instance, we know only conic reflectors provide single viewpoint catadioptric systems. Thus, one can use only elliptical, parabolic or hyperbolic sensors. Also, such imaging systems require precise alignment of the various imaging components. Any miss-alignment, and the system deviates strongly from a single viewpoint.

If we move away from the single viewpoint constraint, then we have much more flexibility in designing imaging systems. For instance, we are no longer limited to using only conic reflectors. More exotic reflectors could be used to develop new sensors. This paper presents a step towards understanding such non-single viewpoint imaging systems. We focussed on conic reflectors not only because most catadioptric systems utilize such reflectors but also because locally any surface can be approximated by quadrics.

We derived a concise analytic expression for the loci of viewpoints (caustics) for catadioptric imaging systems utilizing conic reflectors. In such sensors, every pixel in such systems has an unique viewpoint. Using the framework of caustics, the viewpoint surface can be parameterized with a small number of parameters. These parameters depend on the surface geometry of the reflector and the relative location of the entrance pupil of the lens with respect to the reflector. Under this generic framework, the singleviewpoint catadioptric camera was also shown to be a special case of the general solution. In general, any imaging system, whose components (lenses and reflectors) are completely known, can be replaced by the effective viewpoint surface for the purposes of analysis.

Apart from deriving an analytic expression for the viewpoint surface, we also studied other geometric properties of the sensor. For instance, it was shown that the cusp formed along the optical axis for 
rotationally symmetric systems was closely related to approximating the entire viewpoint surface by a single point. We also proved that the caustic associated with the tangential light ray to the reflector lies at the point of tangentiality. This point "tangent point" was solved for in closed form giving a good bound on the size of the viewpoint surface.

An important reason to stray away from a single viewpoint is to design imaging systems with specific resolution characteristics. We provide a simple analytic study of resolution for such camera systems. We showed how resolution degrades radially beyond some distance from the image center, if the effective viewpoint locus is not a single point. However, careful design of the imaging system in terms of lens and reflector choice and their relative positioning, can be used to exploit the initial rise or plateau effect on resolution. Further the analytic form for resolution can used to optimize the design parameters of the imaging system for apriori resolution requirements.

Finally, we presented a simple calibration technique to estimate the viewpoint surface and camera parameters for a conic catadioptric system using known camera motion. In general the problem of calibrating non-single viewpoint cameras is that of estimating a map between each pixel in the image and its unique viewpoint and viewing direction. Using the framework of caustics this potentially hard problem was reduced to that of estimating a small number of model parameters. Although we assumed knowledge of the reflector shape (conic cross-section), the precise geometry of the reflector and camera as a whole was unknown and was estimated from two images of an arbitrary scene. 


\section{Appendix A}

We now derive the caustic surface for a parameterized conic reflector given by:

$$
A z^{2}+\gamma^{2}+B z=C
$$

This parameterization not only includes parabolic $(c=0, A=0)$, elliptic $(B=0)$ and hyperbolic $(A<0, C<0)$ reflectors, but also spherical $\left(A=1, C+\frac{B^{2}}{4}>0\right)$. As opposed to Eq.2 which didn't model spherical reflectors, Eq.40 includes the sphere as well.

In parametric form, Eq. 40 can be rewritten as:

$$
\begin{aligned}
& z(t)=t \\
& \gamma(t)=\sqrt{C-B t-A t^{2}}
\end{aligned}
$$

Using the Jacobian method [7] we derive the caustic profile as a parametric curve:

$$
\begin{aligned}
S c N x(t)= & B^{3} C^{2}+6 B^{2} C d-4 B C^{2} d+8 B d^{2}-8 C d^{2}-3\left(B-2 A^{2} C\right)\left(B^{2} C+4 B d-4 C d\right) t+ \\
& 6\left(B^{3}-2\left(1+A^{2}\right) B^{2} C+2 A^{2}\left(1+A^{2}\right) B C^{2}+4 A^{2}\left(A^{2}-1\right) C d\right) t^{2}+4\left(\left(2 A^{2}-1\right) B^{2},\right. \\
& \left.-A^{2}\left(1+3 A^{2}\right) B C+2 A^{4}\left(1+A^{2}\right) C^{2}+4 A^{2}\left(A^{2}-1\right) d\right) t^{3} \\
S c N y(t)= & 4\left(B^{2}-4 B C-4 d+4 A^{2}\left(C^{2}+d\right)\right)\left(d-t\left(B+A^{2} t\right)\right)^{(3 / 2)} \\
S c D(t)= & -B^{3} C+4 B^{2} C^{2}+2 B^{2} d-4 B C d+16 A^{2} C^{2} d-8 d^{2}+16 A^{2} d^{2} \\
& -3\left(B^{3}+2\left(A^{2}-2\right) B^{2} C+4\left(2 A^{2}-1\right) B d-8 A^{2} C d\right) t \\
& -12 A^{2}\left(A^{2}-1\right)(B C+2 d) t^{2}+4 A^{2}\left(A^{2}-1\right)\left(B-2 A^{2} C\right) t^{3} \\
S c^{\prime}(t)= & \left\{\frac{S x N x(t)}{S c D(t)}, \frac{S x N y(t)}{S c D(t)}\right\} .
\end{aligned}
$$

Equation (42) describes the caustic profile for the complete class of conic (including spherical) catadioptric cameras. In calibrating such non-single viewpoint imaging systems, when the reflector shape is not known, we should use the above derived model. 


\section{Acknowledgements}

This work was supported in parts by a National Science Foundation ITR Award, IIS-00-85864, and the DARPA Human Indentification (HID) Program, Contract No N00014-00-1-0929.

The authors would like to acknowledge Prof. P.J. Giblin of University of Liverpool for his help through many email discussions. We would also like to thank Srinivas N. and Yoav S. for reading through early drafts of this paper and for their many indispensable suggestions.

\section{References}

[1] S. Baker and S. K. Nayar. A Theory of Catadioptric Image Formation. In Proc. ICCV, pages 35-42, 1998.

[2] S. Baker and S. K. Nayar. A Theory of Single-Viewpoint Catadioptric Image Formation. IJCV, 35(2):1-22, November 1999.

[3] S. Bogner. Introduction to Panoramic Imaging. In IEEE SMC Conference, volume 54, pages 3100-3106, October 1995.

[4] R. C. Bolles, K. G. Konolige, and M. A. Fischler. Extra Set of Eyes. In DARPA-IUW, pages 41-44, 1997.

[5] M. Born and E. Wolf. Principles of Optics. Permagon Press, 1965.

[6] J. W. Bruce, P. J. Giblin, and C. G. Gibson. On Caustics of Plane Curves. American Mathematical Monthly, 88:651-667, November 1981.

[7] D. G. Burkhard and D. L. Shealy. Flux Density for Ray Propoagation in Gemoetrical Optics. Journal of the Optical Societyu of America, 63(3):299-304, March 1973.

[8] J. Chahl and M. Srinivasan. Reflective surfaces for panoramic imaging. Applied Optics, 36(31):8275-8285, 1997.

[9] J. Charles, R. Reeves, and C. Schur. How to build and use an all-sky camera. Astronomy Magazine, April 1987.

[10] S. Derrien. and K. Konolige. Aproximating a single viewpoint in panoramic imaging devices. In International Conference on Robotics and Automation, pages 3932-3939, 2000.

[11] C. Geyer and K. Daniilidis. Catadioptric camera calibration. In Proc. ICCV, pages 398-404, 1999. 
[12] J. Gluckman and S. K. Nayar. Planar Catadioptric Stereo: Geometry and Calibration. In CVPR, pages I:22-28, 1999.

[13] M. D. Grossberg and S. K. Nayar. A General Imaging Model and a Method for Finding its Parameters. In Proc. ICCV, 2001.

[14] W. R. Hamilton. Theory of Systems of Rays. Transactions of the Royal Irish Academy, 15:69-174, 1828.

[15] R. Hicks and R. Bajcsy. Catadioptric Sensors that Approximate Wide-Angle Perspective Projections. In Proc. CVPR, pages I:545-551, 2000.

[16] J. W. Hong, X. Tan, B. Pinette, R. Weiss, and E. M. Riseman. Image-Based Navigation Using 360 Views. In DARPA-IUW, pages 782-791, 1990.

[17] H. W. Jensen. In http://graphics.stanford.edu/ henrik/images/caustics.html, 1996.

[18] S. B. Kang. Catadioptric self-calibration. In Proc. CVPR, pages I:201-207, June 2000.

[19] J. Murphy. Application of panoramic imaging to a teleoperated lunar rover. In IEEE SMC Conference, volume 36, pages 3117-3121, October 1997.

[20] V. Nalwa. A True Omnidirectional Viewer. Technical report, Bell Laboratories, Holmdel, NJ 07733, U.S.A., February 1996.

[21] S. K. Nayar. Catadioptric Omnidirectional Cameras. In Proc. CVPR, pages 482-488, 1997.

[22] S. K. Nayar and A. D. Karmarkar. 360 x 360 Mosaics. In Proc. CVPR, pages I:388-395, 2000.

[23] S. Peleg, Y. Pritch, and M. Ben-Ezraet. Cameras for stereo panoramic imaging. In Proc. CVPR, pages I:208-214, June 2000.

[24] V. N. Peri and S. K. Nayar. Generation of Perspective and Panoramic Video from Omnidirectional Video. DARPA-IUW, pages I:243-245, December 1997.

[25] D. Rees. Panoramic television viewing system. United States Patent No.3,505,465, April 1970.

[26] Y. Yagi, S. Kawato, and S. Tsuji. Real-Time Omnidirectional Image Sensor (copis) for Vision-Guided Navigation. Robotics and Automation, 10(1):11-22, February 1994.

[27] Y. Yagi and M. Yachida. Real-Time Generation of Environmental Map and Obstacle Avoidance Using Omnidirectional Image Sensor with Conic Mirror. In Proc. CVPR, pages 160-165, 1991. 
[28] K. Yamazawa, Y. Yagi, and M. Yachida. Omnidirectional imaging with hyperboloidal projection. In Proc. IEEE/RSJ International Conference on Intelligent Robots and Systems, pages 1029-1034, July 1993. 